\title{
Phase ambiguity of the measure for continuum Majorana fermions
}

\author{
Maarten Golterman ${ }^{1,2}$ and Yigal Shamir ${ }^{3}$ \\ ${ }^{1}$ Department of Physics and IFAE-BIST, Universitat Autònoma de Barcelona E-08193 Bellaterra, \\ Barcelona, Spain \\ ${ }^{2}$ Department of Physics and Astronomy, San Francisco State University, \\ San Francisco, California 94132, USA \\ ${ }^{3}$ Raymond and Beverly Sackler School of Physics and Astronomy, Tel Aviv University, \\ 69978, Tel Aviv, Israel
}

(Received 20 May 2019; published 9 August 2019)

\begin{abstract}
Integrating over a continuum Majorana fermion formally yields a functional Pfaffian. We show that the phase of this Pfaffian is ambiguous, as it depends on the choice of basis. This ambiguity is naturally resolved within a nonperturbative lattice definition, allowing us to discuss the relation between the phase of the lattice Pfaffian and the effective $\theta$ angle of the theory. We also resolve an apparent paradox regarding the induced $\theta$ angle when a theory of $N$ Dirac fermions in a real representation of the gauge group is reexpressed in terms of $2 N$ Majorana fermions. We discuss how all this is reflected in chiral perturbation theory.
\end{abstract}

DOI: 10.1103/PhysRevD.100.034507

\section{INTRODUCTION}

In a QCD-like theory with Dirac fermions, the measure of the Euclidean functional integral is positive when all fermions have a positive mass, and, as a consequence, there is no topological term induced by the fermionic part of the theory. This generalizes to all types of fermion irreducible representations (irreps): complex, real, and pseudoreal.

If the theory contains $N$ Dirac fermions in a real irrep, we may reformulate it in terms of $2 \mathrm{~N}$ Majorana fermions. We will be using Majorana fields each of which packs together a Weyl fermion and its antifermion. ${ }^{1}$ Assuming an equal positive mass $m>0$ for all Dirac flavors, the mass matrix $M$ of the Majorana formulation is then given by $M=m J_{S}$, with the $2 N \times 2 N$ matrix

$$
J_{S}=\left(\begin{array}{cc}
0 & \mathbf{1}_{N} \\
\mathbf{1}_{N} & 0
\end{array}\right)
$$

where $\mathbf{1}_{n}$ is the $n \times n$ unit matrix. A nonanomalous chiral rotation can then be used to bring the mass matrix to a flavor-diagonal form $M=m J_{S}^{\text {rot }}$ where

$$
J_{S}^{\mathrm{rot}}=i \gamma_{5} \mathbf{1}_{2 N}
$$

\footnotetext{
${ }^{1}$ The precise definition is given in Eq. (2.8) below.
}

Published by the American Physical Society under the terms of the Creative Commons Attribution 4.0 International license. Further distribution of this work must maintain attribution to the author(s) and the published article's title, journal citation, and DOI. Funded by SCOAP ${ }^{3}$. showing that each entry of $M$ has a $U(1)_{A}$ phase equal to $\pi / 2$. Now let us apply a $U(1)_{A}$ rotation that turns the mass matrix into a positive matrix, $M=m \mathbf{1}_{2 N}$. Because of the anomaly, this generates a topological term $e^{i \theta Q}$, where

$$
Q=\frac{g^{2}}{32 \pi^{2}} \int d^{4} x \operatorname{tr}(F \tilde{F})
$$

is the topological charge, and

$$
\theta=-\pi N T / 2,
$$

where $T$ is the index of the Dirac operator for the fermion irrep in a single instanton background.

Let us consider the consequences of this topological term. $T$ is always even for a real irrep. ${ }^{2}$ If $N T$ is divisible by 4 then $e^{i \theta Q}=1$, and the topological term drops out. If $N T$ is not divisible by 4 , we have $e^{i \theta Q}=(-1)^{Q}$. Hence, it appears that the Majorana measure will be positive for even $Q$, but negative for odd $Q$. This is puzzling, because the measure of the original Dirac theory is positive for any $Q$, and, obviously, the Dirac and Majorana formulations should represent the same theory.

The paradox would be resolved if the very transition to the Majorana formulation would somehow generate a "compensating" topological term $e^{i \pi N T Q / 2}$. The additional topological term induced by the $U(1)_{A}$ rotation would then cancel against the compensating topological term. We would

\footnotetext{
${ }^{2} \mathrm{We}$ will recover this result in Sec. II.
} 
end up with a positive mass matrix and with no topological term, as in the original Dirac theory.

The purpose of this paper is to show that this is indeed what happens. In reality, it turns out that the paradox described above arises because in the argument we ignored a phase ambiguity of the Majorana measure which is present in the formal continuum theory. The existence of this ambiguity allows us to require agreement between the Dirac and Majorana formulations. When the Majorana mass matrix involves $J_{S}$ or $J_{S}^{\text {rot }}$, this requirement implies the existence of the compensating topological term in the path integral. Going beyond formal arguments, we demonstrate the presence of the compensating topological term through a fully nonperturbative lattice derivation of the transition from the Dirac to the Majorana formulation. Finally, we discuss the implications for the chiral effective theory.

This paper is organized as follows. In Sec. II we show how, in the continuum, a phase ambiguity arises in the choice of a basis for a gauge theory with Majorana fermions. We explain how this ambiguity can be resolved in a theory with an even number of Majorana fermions by comparison with the same theory formulated in terms of Dirac fermions. Then, in Sec. III, we show that the lattice formulation implies a natural choice of basis, thus fixing the phase consistently, both in the formulations with Wilson and with domain-wall fermions. This allows us to discuss the $\theta$ angle induced by the lattice fermion action, reviewing and generalizing the earlier work of Ref. [1]. We consider separately a standalone gauge theory of Majorana fermions, and a theory of $2 N$ Majorana fermions obtained by reformulating a theory of $N$ Dirac fermions. We then revisit the precise form of the condensate in the presence of a fermion-induced $\theta$ angle, both in the gauge theory as well as in chiral perturbation theory. This is done in Sec. IV for a theory with Dirac fermions in a complex irrep of the gauge group, and in Sec. V for a theory with Majorana fermions in a real irrep of the gauge group. Section VI contains our summary and conclusion. There are six Appendixes dealing with technical details.

\section{MAJORANA FERMIONS AND THE PHASE AMBIGUITY}

In this section, we first review some useful standard results for Dirac (Sec. II A) and Majorana (Sec. II B) fermions. We then discuss the phase ambiguity that is encountered in defining the continuum path integral for Majorana fermions (Sec. II C).

\section{A. Dirac fermions}

Consider a Euclidean gauge theory with $N$ Dirac fermions in some irrep of the gauge group. The partition function for the most general choice of parameters is

$$
Z=\int \mathcal{D} A \mathcal{D} \psi \mathcal{D} \bar{\psi} \exp \left(-\int d^{4} x \mathcal{L}\right)
$$

where

$$
\mathcal{L}=\frac{1}{4} F^{2}+\bar{\psi}\left(\not D+\mathcal{M}^{\dagger} P_{L}+\mathcal{M} P_{R}\right) \psi+i \theta Q,
$$

with $P_{R, L}=\left(1 \pm \gamma_{5}\right) / 2$, and $\mathcal{M}$ is a complex $N \times N$ matrix. The topological charge $Q$ was introduced in Eq. (1.3). We will specialize to a mass matrix of the form

$$
\mathcal{M}=m \Omega=m e^{i \alpha /(N T)} \tilde{\Omega}, \quad \tilde{\Omega} \in S U(N),
$$

with real $m>0$ and a real phase $\alpha$. Upon integrating out the fermions the dependence on $\tilde{\Omega}$ drops out thanks to the invariance under nonsinglet chiral transformations, and

$$
\operatorname{det}\left(\not D+\mathcal{M}^{\dagger} P_{L}+\mathcal{M} P_{R}\right)=e^{i \alpha Q} \operatorname{det}^{N}(\not D+m),
$$

where, on the right-hand side, $D+m$ is the one-flavor Dirac operator. This result can be derived using the spectral representation of the Dirac operator; see Appendix A. As mentioned earlier, $T$ is the index of the Dirac operator in a single instanton background. The measure $\mu(A)$ of the path integral is thus

$$
\begin{aligned}
\mu(A) & =e^{-i \theta_{\text {eff }} Q} e^{-\frac{1}{4} F^{2}} \operatorname{det}^{N}(\not D+m) \\
& =e^{-i \theta_{\text {eff }} Q} \tilde{\mu}(A),
\end{aligned}
$$

where

$$
\tilde{\mu}(A)=e^{-\frac{1}{4} F^{2}} \operatorname{det}^{N}(D+m),
$$

is positive, and the effective topological angle is

$$
\theta_{\text {eff }}=\theta-\alpha \text {. }
$$

\section{B. Majorana fermions}

A theory of $N$ Dirac fermions in a real representation of the gauge group $G$ can be reformulated in terms of $2 N$ Majorana fermions. The $N$ Dirac fermions are composed of $2 N$ Weyl fermions. From these Weyl fermions, we construct Majorana fermions each of which packs together a Weyl fermion and its antifermion, which is possible because the fermion and the antifermion belong to the same representation of $G$.

The mapping between Dirac fermions (on the right-hand side) and Majorana fermions (on the left-hand side) is

$$
\begin{aligned}
\Psi_{L, i} & =\psi_{L, i}, \\
\Psi_{R, i} & =C S \bar{\psi}_{L, i}^{T}, \\
\Psi_{R, N+i} & =\psi_{R, i}, \\
\Psi_{L, N+i} & =C S \bar{\psi}_{R, i}^{T},
\end{aligned}
$$


where $i=1, \ldots, N$. Here $C$ is the charge-conjugation matrix, and $S$ is the group tensor satisfying the invariance property $g^{T} S g=S$ for all $g \in G$. We recall the basic properties, $C^{-1}=C^{\dagger}=C^{T}=-C$, and $S^{-1}=S^{\dagger}=S^{T}=S$. We also introduce

$$
\bar{\Psi} \equiv \Psi^{T} C S
$$

Thus, Eq. (2.8) determines all the components of the Majorana fermions in terms of the original Dirac fermions, or, equivalently, in terms of the corresponding Weyl fields. Other mappings between Dirac and Majorana fermions are possible, and we give an example in Appendix B. What is special about Eq. (2.8) is that it respects the natural mapping between Weyl and Majorana fields.

Proceeding to the lagrangian, for the kinetic term we have

$$
\mathcal{L}_{K}=\sum_{i=1}^{N} \bar{\psi} \not \partial \psi=\frac{1}{2} \sum_{I=1}^{2 N} \bar{\Psi}_{I} \not \Psi_{I} .
$$

For the mass term we have

$$
\mathcal{L}_{m}=m \bar{\psi} e^{i \alpha_{D} \gamma_{5}} \psi=\frac{m}{2} \bar{\Psi} e^{i \alpha_{D} \gamma_{5}} J_{S} \Psi,
$$

where the $2 N \times 2 N$ matrix $J_{S}$ was introduced in Eq. (1.1), and $\alpha_{D}=\alpha /(N T)$ is the phase introduced in the Dirac case in Eq. (2.3). We have set $\tilde{\Omega}=1$, since the $S U(N)$ part of the original Dirac mass matrix does not play a role in the following.

The flavor symmetry is as follows. In the massless limit, the theory is invariant under $S U(2 N)$ transformations

$$
\begin{aligned}
& \Psi \rightarrow\left(P_{L} h+P_{R} h^{*}\right) \Psi, \\
& \bar{\Psi} \rightarrow \bar{\Psi}\left(P_{L} h^{T}+P_{R} h^{\dagger}\right),
\end{aligned}
$$

with $h \in S U(2 N)$. When the mass term (2.11) is turned on, the $S U(2 N)$ symmetry is explicitly broken to $S O(2 N)$. The Dirac formulation of the same theory obviously has the same global symmetry, but the full symmetry is manifest only in the Majorana formulation. ${ }^{3}$

\section{Pfaffian phase ambiguity}

There exists a nonanomalous $S U(2 N)$ chiral rotation that brings the Majorana mass term (2.11) to a diagonal form

$$
\mathcal{L}_{m}=\frac{m}{2} \bar{\Psi} i \gamma_{5} e^{i \alpha_{D} \gamma_{5}} \Psi=\frac{m}{2} \bar{\Psi} e^{i\left(\alpha_{D}+\pi / 2\right) \gamma_{5}} \Psi .
$$

We see that we have an extra $U(1)$ phase of $\pi / 2$, leading to an apparent paradox, as explained in the Introduction.

\footnotetext{
${ }^{3}$ For a discussion of how the global symmetry is realized in the Dirac formulation, see Ref. [2].
}

In the following, we ask the question of how this paradox may be resolved in the continuum. In Sec. III we will show how it is avoided, by introducing a nonperturbative regulator.

To start, let us consider a single Majorana fermion with Lagrangian

$$
\begin{gathered}
\mathcal{L}=\frac{1}{2} \bar{\Psi} D \Psi=\frac{1}{2} \Psi^{T} C S D \Psi \\
D=\not D+m e^{i \alpha_{M} \gamma_{5}} .
\end{gathered}
$$

The differential operator CSD is antisymmetric, and the result of formally integrating out the Majorana fermion is $\operatorname{pf}(C S D)$, the Pfaffian of $C S D$.

In the Dirac case, $\operatorname{det}(D)$ is simply equal to the (regulated) product of all eigenvalues; see Appendix A. What about Pfaffians?

Introducing the abbreviation $\mathcal{A}=C S D$, the effect of a unitary change of basis for Majorana fermions is

$$
\mathcal{A} \rightarrow \mathcal{A}^{\prime}=\mathcal{U}^{T} \mathcal{A} \mathcal{U}
$$

where $\mathcal{A}$ and thus $\mathcal{A}^{\prime}$ are antisymmetric. We will be looking for a change of basis so that $\mathcal{A}^{\prime}$ will have a skewdiagonal form.

For a real representation, the eigenvalues of the Dirac operator have a twofold degeneracy. Because its Hermitian part is equal to $m \cos \alpha_{M}$ times the identity matrix, the Dirac operator (2.15) is normal, $\left[D, D^{\dagger}\right]=0$. Consider an eigenvector $\chi$ with eigenvalue $\lambda$. By normality, $D \chi=\lambda \chi$ implies $D^{\dagger} \chi=\lambda^{*} \chi$. Hence

$$
D C S \chi^{*}=C S D^{T} \chi^{*}=C S\left(D^{\dagger} \chi\right)^{*}=C S\left(\lambda^{*} \chi\right)^{*}=\lambda C S \chi^{*} .
$$

It follows that $C S \chi^{*}$ is an eigenmode with the same eigenvalue as $\chi$. The eigenmodes $\chi$ and $C S \chi^{*}$ are orthogonal, $\left(C S \chi^{*}\right)^{\dagger} \chi=-\chi^{T} C S \chi=0$, where we used that the matrix $C S$ is antisymmetric.

The skew-diagonal representation $\mathcal{A}^{\prime}$ is achieved by transforming to a basis in which each eigenvector $\chi$ is followed by its companion eigenvector $C S \chi^{*}$. Selecting arbitrarily one eigenvector from each pair, and labeling the resulting subset as $\chi_{1}, \chi_{2}, \ldots$, we consider the unitary change of basis generated by the matrix $\mathcal{U}$ whose columns are comprised of the ordered pairs of eigenvectors,

$$
\mathcal{U}=\left(\chi_{1}, e^{i \phi_{1}} C S \chi_{1}^{*}, \chi_{2}, e^{i \phi_{2}} C S \chi_{2}^{*}, \ldots\right) .
$$

Notice that, for each pair, we have allowed the second eigenvector to have an arbitrary $\mathrm{U}(1)$ phase relative to the original eigenvector. These arbitrary phases play a profound role, as we will now see. 
The $2 \times 2$ subspace of $\mathcal{A}^{\prime}$ associated with a pair $\chi, e^{i \phi} C S \chi^{*}$ with eigenvalue $\lambda$ has the explicit form

$$
\left(\begin{array}{c}
\chi^{T} \\
e^{i \phi} \chi^{\dagger} S C^{T}
\end{array}\right) C S D\left(\chi e^{i \phi} C S \chi^{*}\right)=e^{i \phi} \lambda\left(\begin{array}{cc}
0 & -1 \\
1 & 0
\end{array}\right) .
$$

The Pfaffian of $\mathcal{A}^{\prime}$ factorizes as the product of Pfaffians for the $2 \times 2$ subspaces, where the Pfaffian of the above $2 \times 2$ subspace is, by definition, equal to $-e^{i \phi} \lambda$. Explicitly,

$$
\operatorname{pf}\left(\mathcal{A}^{\prime}\right)=\prod_{i}\left(-e^{i \phi_{i}} \lambda_{i}\right)
$$

This result exhibits a phase ambiguity, represented by the $\operatorname{sum} \sum_{i} \phi_{i}$.

In retrospect, the phase ambiguity can be traced to the elementary property $\operatorname{pf}\left(\mathcal{A}^{\prime}\right)=\operatorname{pf}(\mathcal{A}) \operatorname{det}(\mathcal{U})$. This relation implies that the phase of the Pfaffian depends on the choice of basis for the Majorana field on which the differential operator $\mathcal{A}$ acts. The basis is represented by the unitary matrix $\mathcal{U}$, and $\operatorname{det}(\mathcal{U})$ is, thus, a basis-dependent phase.

The rigorous resolution of the phase ambiguity requires a nonperturbative treatment in order to specify the basis, which we will give in Sec. III. In the rest of this section we restrict ourselves to an even number of Majorana fermions, and discuss how the phase may be fixed by appealing to the corresponding theory defined in terms of Dirac fermions, where no such phase ambiguity exists.

As reviewed in Appendix A for the Dirac case, let us consider separately the zero modes and the nonzero modes. Starting with the nonzero modes, and following Appendix A, the eigenvectors $\chi_{ \pm}$now each have a companion, $e^{i \phi_{ \pm}} C S \chi_{ \pm}^{*}$, where we have allowed for arbitrary relative $\mathrm{U}(1)$ phases. The contribution of these two pairs of eigenvectors to $\operatorname{pf}(\mathcal{A})$ is

$$
\left(-e^{i \phi_{+}} \lambda_{+}\right)\left(-e^{i \phi_{-}} \lambda_{-}\right)=e^{i\left(\phi_{+}+\phi_{-}\right)}\left(\lambda^{2}+m^{2}\right),
$$

where, as in Appendix A, $\lambda^{2}$ is the eigenvalue of the secondorder operator $-\not^{2} P_{R}$. For a single Dirac fermion in the same real representation, the contribution of the eigenvectors $\chi_{ \pm}$and $e^{i \phi_{ \pm}} C S \chi_{ \pm}^{*}$ to $\operatorname{det}(D)$ is simply a factor of

$$
\left(\lambda^{2}+m^{2}\right)^{2}
$$

The determinant is independent of the arbitrary U(1) phase of each eigenvector. If we now take two Majorana fermions, the corresponding contribution to $\operatorname{pf}\left(\mathcal{A}^{\prime}\right)$ will be

$$
\left[e^{i\left(\phi_{+}+\phi_{-}\right)}\left(\lambda^{2}+m^{2}\right)\right]^{2} .
$$

We see that, by making the choice

$$
\phi_{+}=\phi_{-}=0,
$$

we achieve agreement between the corresponding factors for the Dirac and two-Majorana cases. ${ }^{4}$

Proceeding to the zero modes, in the Dirac case the contribution of a pair of zero modes, $\chi_{0}, e^{i \phi_{0}} C S \chi_{0}^{*}$, is just

$$
\left(m e^{ \pm i \alpha_{D}}\right)^{2}
$$

depending on the chirality. In the Majorana case, the corresponding contribution to $\operatorname{pf}\left(\mathcal{A}^{\prime}\right)$ from each Majorana fermion is

$$
-m e^{i \phi_{0}} e^{ \pm i \alpha_{M}}=-m e^{i \phi_{0}} e^{ \pm i\left(\alpha_{D}+\pi / 2\right)}
$$

where on the right-hand side we have substituted $\alpha_{M}=\alpha_{D}+\pi / 2$. The extra phase of $\pi / 2$ arises during the transition from the Dirac to the Majorana formulation, as we have seen in Eq. (2.13). The contribution from two Majorana fermions is thus

$$
\left(m e^{i \phi_{0}} e^{ \pm i\left(\alpha_{D}+\pi / 2\right)}\right)^{2} .
$$

It follows that the Dirac result (2.25) will only be reproduced provided we make the nontrivial choice

$$
\phi_{0}=\pi / 2 \bmod \pi .
$$

\section{NONPERTURBATIVE CALCULATION}

In the previous section, we showed that the definition of a theory with Majorana fermions has an intrinsic phase ambiguity, which can be used to resolve the apparent paradox introduced in Sec. I. However, the question of whether, and how, the theory "chooses" the proper phase was left open. In order to address this question, we need a properly regulated nonperturbative definition of the theory, which is provided by the lattice.

The lattice action for a Majorana fermion will always have the generic form $\frac{1}{2} \Psi^{T} \mathcal{A} \Psi$ for a suitable antisymmetric operator $\mathcal{A}$. Integrating over the lattice Majorana field yields $\operatorname{pf}(\mathcal{A})$, which is now well defined. There is no room for any (phase) ambiguity, because, on any finite-volume lattice, $\mathcal{A}$ is a finite-size matrix, and the lattice selects the coordinate basis to define $\mathcal{A}$.

Our first result concerns a single Majorana fermion with no chiral angle(s), and a positive bare mass $m_{0}>0$. Using domain-wall fermions, we show in Appendix $\mathrm{C}$ that $\operatorname{pf}(\mathcal{A})$ is strictly positive in this case. The domain-wall fermion measure is then strictly positive for any number of Majorana fermions, and in all topological sectors.

In this section, we discuss in detail the transition from the Dirac to the Majorana formulation. In Sec. III A, we

\footnotetext{
${ }^{4}$ It is, in fact, sufficient to choose $\phi_{+}+\phi_{-}=0 \bmod \pi$.
} 
regulate the theory using Wilson fermions, and in Sec. III B, using domain-wall fermions. While in the case of Wilson fermions, there is a lacuna in the argument (which we discuss in some detail in Appendix D), this is not the case for domain-wall fermions. We establish that the compensating topological term alluded to in the introduction indeed arises when needed, thus resolving the paradox. As in Appendix C, it proves easier to work with the five-dimensional formulation of domain-wall fermions, rather than directly with any Ginsparg-Wilson operator that arises in the limit of an infinite fifth dimension. We also remark that staggered fermions always lead to a fourfold taste degeneracy in the continuum limit, and so they cannot be used here, given that the apparent paradox only arises when $N T$ is even, but not divisible by $4 .{ }^{5}$ We summarize the results of this section in Sec. III C.

\section{A. Wilson fermions}

If we formulate the theory using Wilson fermions, the resolution of the puzzle relies on the observation, made in Ref. [1], of how the $\theta$ angle can be realized within this fermion formulation. The starting point of the discussion is a one-flavor Wilson operator with both the Wilson and mass terms chirally rotated by angles $\theta_{W}$ and $\theta_{m}$, respectively,

$$
D_{W}\left(\theta_{W}, \theta_{m}\right)=D_{K}+e^{i \theta_{W} \gamma_{5}} W+e^{i \theta_{m} \gamma_{5}} m_{0} .
$$

Here $D_{K}$ is the naive lattice discretization of the (massless) Dirac operator. $W$ is the Wilson term, which eliminates the fermion doublers, and is chosen for definiteness to be real and positive; $m_{0}$ is the bare mass. The partition function takes the form (2.1), but with the fermion part of the Lagrangian replaced by ${ }^{6}$

$$
\mathcal{L}_{F}=\bar{\psi} D_{W}\left(\theta_{W}, \theta_{m}\right) \psi .
$$

First, only the difference $\theta_{W}-\theta_{m}$ can be physical, as can be seen by applying the transformation

$$
\psi \rightarrow e^{i \eta \gamma_{5}} \psi, \quad \bar{\psi} \rightarrow \bar{\psi} e^{i \eta \gamma_{5}} .
$$

In the lattice-regulated theory, the determinant of this transformation is unity, and hence it provides an alternative representation of exactly the same theory. It is easily checked that this transformation leaves the $D_{K}$ part invariant, while the angles undergo the transformation $\theta_{W} \rightarrow \theta_{W}+2 \eta$, $\theta_{m} \rightarrow \theta_{m}+2 \eta$. By choosing $\eta=-\theta_{m} / 2$ we eliminate the phase of the mass term, while the phase of the Wilson term becomes $\theta_{F} \equiv \theta_{W}-\theta_{m}$.

With only the angle $\theta_{F}$ left in the fermion action, and with $\theta$ as the explicit vacuum angle [see Eq. (2.2)], what Ref. [1] claimed is that, in the continuum limit,

\footnotetext{
${ }^{5}$ Interpreting the staggered tastes as physical flavors, it is possible that reduced staggered fermions can be employed [3,4]. We have not explored this further.

${ }^{6} \mathrm{We}$ will not need the lattice form of the gauge action.
}

$$
Z\left(\theta, \theta_{F}\right)=Z\left(\theta+N T \theta_{F}, 0\right)
$$

This implies that the relative $U(1)$ phase of the Wilson term and the mass term turns into the familiar $\theta$ angle in the continuum limit. In Eq. (3.4) we have written down the generalization of the result of Ref. [1] to $N$ Dirac fermions in an irrep with index $T$. In the case that a topological term with $\theta \neq 0$ is already present in the gauge action, $N T \theta_{F}$ gets added to $\theta$.

We pause here to note that the argument given in Ref. [1] is not complete as it stands, because of a subtlety related to renormalization. While it is beyond the scope of this paper to complete the proof, Appensdix D outlines a conjecture on the interplay of the observation of Ref. [1] and renormalization. However, this subtlety does not affect the rest of this paper. In particular, in the next subsection we provide an argument analogous to the one given here based on domain-wall fermions, where the subtlety does not arise.

Next, let us work out the transition from the Dirac to the Majorana case. We start with a single Dirac fermion in a real irrep, where the Wilson fermion operator $D_{W}\left(\theta_{F}\right)$ is given by Eq. (3.1), taking $\theta_{W}=\theta_{F}$ and $\theta_{m}=0$. In the Majorana formulation, the $4 \times 4$ matrix in spinor space becomes an $8 \times 8$ matrix which mixes the two Majorana species. In terms of $4 \times 4$ blocks, the Wilson operator in the Majorana formulation is

$$
D_{\mathrm{Maj}}\left(\theta_{F}\right)=\left(\begin{array}{cc}
D_{K} & e^{i \theta_{F} \gamma_{5}} W+m_{0} \\
e^{i \theta_{F} \gamma_{5}} W+m_{0} & D_{K}
\end{array}\right),
$$

where we have used Eqs. (2.8) and (2.9). The Lagrangian becomes

$$
\mathcal{L}_{F}=\frac{1}{2} \bar{\Psi} D_{\text {Maj }}\left(\theta_{F}\right) \Psi
$$

The key feature of Eq. (3.5) is that, because of their identical chiral properties, the Wilson and mass terms occur in the same places. Applying an $S U(2)$ flavor transformation, i.e., using Eq. (2.12) for $N=1$ with $h=$ $\exp \left(-i \pi \sigma_{2} / 4\right)=h^{*}$, and using that $h^{T} \sigma_{1} h=\sigma_{3}$, the Majorana Wilson operator gets rotated into

$D_{\text {Maj }}\left(\theta_{F}\right)=\left(\begin{array}{cc}D_{K}+e^{i \theta_{F} \gamma_{5}} W+m_{0} & 0 \\ 0 & D_{K}-\left(e^{i \theta_{F} \gamma_{5}} W+m_{0}\right)\end{array}\right)$.

When $\theta_{F}=0$, the relative phase of the Wilson and mass terms is zero, for both of the Majorana species. This implies that $D_{\text {Maj }}(0)$ is the Wilson operator for two Majorana fermions with the same bare mass $m_{0}$ (as opposed to the case where one Majorana fermion would have a mass $+m_{0}$ and the other $-m_{0}$ ). 
We prove this assertion by applying the transformation (3.3) with $\eta=\pi / 2$ to the second Majorana fermion only. ${ }^{7}$ Explicitly, it reads $\Psi_{2} \rightarrow i \gamma_{5} \Psi_{2}$. The Majorana-Wilson operator transforms into

$$
\begin{aligned}
D_{\mathrm{Maj}}\left(\theta_{F}\right) & \\
& \rightarrow\left(\begin{array}{cc}
D_{K}+e^{i \theta_{F} \gamma_{5}} W+m_{0} & 0 \\
0 & i \gamma_{5}\left(D_{K}-\left(e^{i \theta_{F} \gamma_{5}} W+m_{0}\right)\right) i \gamma_{5}
\end{array}\right) \\
& =\left(\begin{array}{cc}
D_{K}+e^{i \theta_{F} \gamma_{5}} W+m_{0} & 0 \\
0 & D_{K}+e^{i \theta_{F} \gamma_{5}} W+m_{0}
\end{array}\right)
\end{aligned}
$$

The fermion operator for each Majorana fermion is now exactly the same as in the Dirac case. The corresponding basis for the Majorana fields is given in Appendix B. It follows that the fermion measure of the two-Majorana formulation is equal to $\operatorname{pf}^{2}\left(\operatorname{CSD}_{W}\left(\theta_{F}\right)\right)$, and thus equal to the Dirac measure $\operatorname{det}\left(D_{W}\left(\theta_{F}\right)\right)$. We have proved that the fermion measure in the Majorana formulation is unchanged from the Dirac formulation.

Equation (3.8) shows that we can choose the mass matrix to be proportional to the unit matrix, instead of to $J_{S}$ [Eq. (1.1)] or $J_{S}^{\text {rot }}$ [Eq. (1.2)]. Unlike in the formal continuum treatment of the previous section, no phase ambiguity, nor any "excess" phase of $\pi / 2$, arises when the transition to Majorana fermions is done in the lattice-regulated theory.

\section{B. Domain-wall fermions}

In this subsection, we revisit the argument of the previous subsection, but now using domain-wall fermions [5] instead of Wilson fermions. As we will see, in the case of domainwall fermions, the argument is complete, allowing us to conclude that a lattice regularization can indeed be invoked to settle the ambiguity we found in Sec. II.

The starting point is the domain-wall fermion action [6] for a massive Dirac fermion with bare mass $m_{0}$ and domain-wall height $M$,

$$
\begin{aligned}
S= & \sum_{s=1}^{N_{5}} \bar{\psi}(s)\left(D_{K}+M-1-W\right) \psi(s) \\
& +\sum_{s=1}^{N_{5}-1}\left(\bar{\psi}(s) P_{R} \psi(s+1)+\bar{\psi}(s+1) P_{L} \psi(s)\right) \\
& -m_{0}\left(\bar{\psi}\left(N_{5}\right) P_{R} \psi(1)+\bar{\psi}(1) P_{L} \psi\left(N_{5}\right)\right),
\end{aligned}
$$

where $\psi$ is the five-dimensional fermion field $\psi(x, s)$, $s=1, \ldots, N_{5}$. In Eq. (3.9), only the dependence on the fifth coordinate is made explicit. The mass term couples the fields on opposite boundaries.

\footnotetext{
${ }^{7}$ Note that the transformation (3.3) is consistent with the Majorana condition (2.9).
}

Domain-wall fermions are not exactly massless for finite $N_{5}$ when $m_{0}=0$. The mass induced by a finite fifth direction, usually referred to as the residual mass, is reminiscent of the additive mass renormalization of Wilson fermions. However, the residual mass vanishes in the limit $N_{5} \rightarrow \infty$, which we will take before the continuum limit. Following this order of limits, the mass term introduced in Eq. (3.9) renormalizes multiplicatively. Thus, the complications of the additive mass renormalization of the Wilson case, that we encountered in Sec. III A, are avoided.

Our aim in this subsection is to recast the argument given in Sec. III A in terms of the domain-wall formulation of the lattice regularized theory. The first step is to prove an analogous result to Eq. (3.4), thus rederiving the theorem of Ref. [1] in terms of domain-wall fermions. For this, we need to define an axial transformation. We take $N_{5}=2 \mathrm{~K}$ even, and define the axial transformation as [7]

$$
\begin{aligned}
& \delta \psi(s)=e^{i \eta} \psi(s), \quad \delta \bar{\psi}(s)=\bar{\psi}(s) e^{-i \eta}, \quad 1 \leq s \leq K, \\
& \delta \psi(s)=e^{-i \eta} \psi(s), \quad \delta \bar{\psi}(s)=\bar{\psi}(s) e^{i \eta}, \quad K+1 \leq s \leq 2 K .
\end{aligned}
$$

Following Ref. [7], we define the five-dimensional currents

$$
\begin{aligned}
j_{\mu}(x, s)= & \frac{1}{2}\left(\bar{\psi}(x, s)\left(1+\gamma_{\mu}\right) U_{\mu}(x) \psi(x+\mu, s)\right. \\
& \left.-\bar{\psi}(x+\mu, s)\left(1-\gamma_{\mu}\right) U_{\mu}^{\dagger}(x) \psi(x, s)\right), \\
j_{5}(x, s)= & \bar{\psi}(x, s) P_{R} \psi(x, s+1)-\bar{\psi}(x, s+1) P_{L} \psi(x, s) .
\end{aligned}
$$

The four-dimensional axial current corresponding to the axial transformation (3.10) is

$$
j_{\mu}^{A}(x)=-\sum_{s=1}^{K} j_{\mu}(x, s)+\sum_{s=K+1}^{2 K} j_{\mu}(x, s) .
$$

It satisfies the Ward-Takahashi identity

$\partial_{\mu}^{-} j_{\mu}^{A}=2 j_{5}(K)+2 m\left(\bar{\psi}(2 K) P_{R} \psi(1)-\bar{\psi}(1) P_{L} \psi(2 K)\right)$,

where $\partial_{\mu}^{-}$is the backward lattice derivative, $\partial_{\mu}^{-} f(x)=$ $f(x)-f(x-\mu)$.

Analogous to Eq. (3.1), we can now introduce two angles, through the combinations $S_{W}\left(\theta_{W}\right)$ and $S_{m}\left(\theta_{m}\right)$, where

$$
\begin{aligned}
S_{W}\left(\theta_{W}\right) & =e^{i \theta_{W}} \bar{\psi}(K) P_{R} \psi(K+1)+e^{-i \theta_{W}} \bar{\psi}(K+1) P_{L} \psi(K), \\
S_{m}\left(\theta_{m}\right) & =-m_{0}\left(e^{-i \theta_{m}} \bar{\psi}(2 K) P_{R} \psi(1)+e^{i \theta_{m}} \bar{\psi}(1) P_{L} \psi(2 K)\right) .
\end{aligned}
$$

$S_{W}\left(\theta_{W}\right)$ replaces the $s=K$ term on the second line of Eq. (3.9), and $S_{m}\left(\theta_{m}\right)$ replaces the mass term (third line) in 
Eq. (3.9). Once again, under an axial transformation [Eq. (3.10)], $\theta_{m, W} \rightarrow \theta_{m, W}+2 \eta$, and hence only the difference $\theta_{F}=\theta_{W}-\theta_{m}$ is physical.

Slightly generalizing the discussion of the previous subsection, here we will keep both $\theta_{W}$ and $\theta_{m}$ arbitrary. If we now differentiate the fermion partition function with respect to $\theta_{W}$, the result is $\left\langle\tilde{j}_{5}\left(\theta_{W}\right)\right\rangle$, where $\langle\cdot\rangle$ indicates integration over the fermion fields, and we have defined

$\tilde{j}_{5}\left(\theta_{W}\right)=e^{i \theta_{W}} \bar{\psi}(K) P_{R} \psi(K+1)-e^{-i \theta_{W}} \bar{\psi}(K+1) P_{L} \psi(K)$.

We will prove that in the theory with a nonzero $\theta_{W}$, the continuum limit of $\left\langle\tilde{j}_{5}\left(\theta_{W}\right)\right\rangle$ yields the axial anomaly. By integrating with respect to $\theta_{W}$, it then follows that

$$
Z\left(\theta, \theta_{W}, \theta_{m}\right)=Z\left(\theta+N T \theta_{W}, 0, \theta_{m}\right),
$$

where now the path integral is defined with the domainwall fermion action instead of the Wilson fermion action, and we have again allowed for $N$ Dirac fermions in an irrep with index $T$. Equation (3.16) generalizes Eq. (3.4) of the preceding subsection.

The proof turns out to be quite straightforward. Let $G\left(\theta_{W}, \theta_{m}\right)$ be the inverse of the domain-wall Dirac operator $D\left(\theta_{W}, \theta_{m}\right)$, with angles $\theta_{W}$ and $\theta_{m}$ introduced as in Eq. (3.14). Using Eq. (3.15), and writing $\tilde{j}_{5}\left(\theta_{W}\right)=$ $\bar{\psi} J_{5}\left(\theta_{W}\right) \psi$, we have

$$
\begin{aligned}
\left\langle\tilde{j}_{5}\left(\theta_{W}\right)\right\rangle & =-\operatorname{Tr}\left(J_{5}\left(\theta_{W}\right) G\left(\theta_{W}, \theta_{m}\right)\right) \\
& =-\operatorname{Tr}\left(J_{5}(0) G\left(0, \theta_{m}-\theta_{W}\right)\right),
\end{aligned}
$$

where in the second step we used the axial transformation (3.10) with $\eta=\theta_{W} / 2$ to move the angle $\theta_{W}$ to the mass term. We now take the limit $K \rightarrow \infty$, in which the propagator in Eq. (3.17) becomes translationally invariant in the fifth dimension. In particular, the propagator becomes independent of the boundaries, and thus of $m$ and $\theta_{m}$ (or $\theta_{m}-\theta_{W}$ after the axial rotation). It follows that $\left\langle\tilde{j}_{5}\left(\theta_{W}\right)\right\rangle=\left\langle\tilde{j}_{5}(0)\right\rangle$ for any $\theta_{W}$ and $\theta_{m}$, and the anomaly is recovered as in Ref. [6].

With the domain-wall equivalent of Eq. (3.4) in hand, we now return to the equivalence between one Dirac fermion in a real irrep of the gauge group and two Majorana fermions, in the domain-wall regularization. As we will see, the argument follows similar steps as that for the Wilsonfermion case given in Sec. III A.

We begin by mapping the action (3.9) into an action for two Majorana fermions, denoted as $\Psi_{i}, i=1,2$. We again make use of Eq. (2.8), but now with a Majorana condition adapted for domain-wall fermions. Analogous to Eq. (2.9), we will require that [8]

$$
\bar{\Psi}=\left(R_{5} \Psi\right)^{T} C S,
$$

with $S$ and $C$ as in Sec. II, and $R_{5}$ is a reflection in the fifth direction:

$$
R_{5} \Psi(x, s)=\Psi\left(x, N_{5}-s+1\right) .
$$

The reason for adding the reflection is that charge conjugation (in four dimensions) interchanges left- and righthanded fermions. Here the right- and left-handed modes emerge near the boundaries $s=1$ and $s=N_{5}$, respectively, and they need to be explicitly interchanged to match the four-dimensional picture. The domain-wall fermion action (3.9) in terms of two massless Majorana fermions $\Psi_{1,2}$ defined by

$\Psi_{L, 1}(s)=\psi_{L}(s)$,

$\Psi_{R, 1}(s)=R_{5} S C \bar{\psi}_{L}^{T}(s)=S C \bar{\psi}_{L}^{T}\left(N_{5}-s+1\right)$,

$\Psi_{L, 2}(s)=R_{5} S C \bar{\psi}_{R}^{T}(s)=S C \bar{\psi}_{R}^{T}\left(N_{5}-s+1\right)$,

$\Psi_{R, 2}(s)=\psi_{R}(s)$,

is then given, for $m_{0}=0$, by

$$
\begin{aligned}
S_{\text {Maj }}= & \frac{1}{2} \sum_{s=1}^{N_{5}} \Psi^{T}\left(N_{5}-s+1\right) C S D_{K} \Psi(s) \\
& +\frac{1}{2} \sum_{s=1}^{N_{5}} \Psi^{T}\left(N_{5}-s+1\right) C S \sigma_{1}(M-W-1) \Psi(s) \\
& +\frac{1}{2} \sum_{s=1}^{N_{5}-1}\left(\Psi^{T}\left(N_{5}-s+1\right) C S \sigma_{1} P_{R} \Psi(s+1)\right. \\
& \left.+\Psi^{T}\left(N_{5}-s\right) C S \sigma_{1} P_{L} \Psi(s)\right)
\end{aligned}
$$

where $\sigma_{1}$ is again the first Pauli matrix acting on the flavor index $i=1,2$ of $\Psi_{i}$. Using Eq. (3.19) and Eq. (3.20), the Majorana form of Eq. (3.14) is

$$
\begin{aligned}
S_{W}\left(\theta_{W}\right)= & \frac{1}{2}\left(e^{i \theta_{W}} \Psi_{R}^{T}(K+1) S C \sigma_{1} \Psi_{R}(K+1)\right. \\
& \left.+e^{-i \theta_{W}} \Psi_{L}^{T}(K) S C \sigma_{1} \Psi_{L}(K)\right), \\
S_{m}\left(\theta_{m}\right)= & -\frac{m_{0}}{2}\left(e^{-i \theta_{m}} \Psi_{R}^{T}(1) S C \sigma_{1} \Psi_{R}(1)\right. \\
& \left.+e^{i \theta_{m}} \Psi_{L}^{T}(2 K) S C \sigma_{1} \Psi_{L}(2 K)\right) .
\end{aligned}
$$

$S_{m}\left(\theta_{m}\right)$ gets added to the massless Majorana domain-wall action (3.21), while $S_{W}\left(\theta_{W}\right)$ replaces the $s=K$ term in the third and fourth lines of Eq. (3.21).

As in Sec. III A, the flavor matrix $\sigma_{1}$ in Eq. (3.22) can be rotated into $\sigma_{3}$. If we then perform a phase transformation ${ }^{8}$

\footnotetext{
${ }^{8}$ Again, this phase transformation is not anomalous on the lattice.
} 


$$
\begin{array}{ll}
\Psi_{2}(x, s) \rightarrow i \Psi_{2}(x, s), & 1 \leq s \leq K, \\
\Psi_{2}(x, s) \rightarrow-i \Psi_{2}(x, s), & K+1 \leq s \leq 2 K,
\end{array}
$$

on the Majorana field $\Psi_{2}$, while leaving $\Psi_{1}$ alone, this rotates $\sigma_{3}$ into the identity matrix in flavor space. The end result is that $\sigma_{1}$ is removed from Eqs. (3.21) and (3.22) (while leaving the kinetic term invariant), thus proving that the theory has two Majorana fermions with equal positive mass $m$ and the same $\theta$ angle as the Dirac theory. Again, using that $\mathrm{pf}^{2}(\mathcal{A})=\operatorname{det}(\mathcal{A})$ for any antisymmetric $\mathcal{A}$, we conclude that the Majorana measure is identical to the Dirac measure.

\section{Summary}

We summarize the main results of this section. The starting point is a lattice-regularized theory with Wilson or domainwall fermions, and with chiral angles $\theta_{m}$ and $\theta_{W}$ introduced in Eqs. (3.1) or (3.14), respectively. ${ }^{9}$ We also allow for an explicit topological term in the gauge action, with angle $\theta$ [see Eq. (2.2)].

Consider first the case of $N$ identical Dirac fermions. As first observed in Ref. [1], in the continuum limit an additional vacuum angle

$$
\theta_{\text {ind }}=N T \theta_{W},
$$

is induced by the fermions. Introducing the "shifted" angle

$$
\theta_{\text {shf }}=\theta+\theta_{\text {ind }},
$$

the operational meaning of this statement is that all observables will be reproduced in the continuum limit if we set $\theta_{W}=0$, and, at the same time, replace $\theta$ by $\theta_{\text {shf }}$ as the angle multiplying the explicit topological term in the (lattice) Lagrangian. As for the phase of the fermion mass matrix, we trivially have $\alpha=N T \theta_{m}$ [recall Eq. (2.3)]. Substituting this into Eq. (2.7) we conclude that, after integrating out the fermions, the effective vacuum angle in the gauge field measure is

$$
\theta_{\mathrm{eff}}=\theta_{\mathrm{shf}}-\alpha=\theta+N T\left(\theta_{W}-\theta_{m}\right) .
$$

In the case of $N_{m a j}$ identical Majorana fermions, the same result holds, with $N=N_{m a j} / 2$.

The interesting case is an even number $2 N$ of Majorana fermions, which we have shown to be equivalent to $N$ Dirac fermions, as they should be. This has resolved the apparent paradox described in Sec. I. We conclude this section by summarizing the result in the case of a single Dirac fermion, $N=1$.

\footnotetext{
${ }^{9}$ Or, to be more precise, in Eq. (D2) in the case of Wilson fermions.
}

The key observation is that, after the transition from a Dirac fermion to two Majorana fermions, the mass term and the Wilson term (or its domain-wall fermion counterpart) are proportional to the same matrix in flavor space. As we have shown, by a sequence of nonanomalous lattice transformations (meaning that the Jacobian of each lattice transformation is equal to one), we may bring the two Majorana fermions to a diagonal form, with the same phases as for the original Dirac fermion [see, e.g., Eq. (3.8) for the Wilson case].

Alternatively, we may elect to apply only SU(2) transformations to the Majorana fermions. These can bring the Wilson and mass terms, that originally point in the $\sigma_{1}$ direction in flavor space, first into the $\sigma_{3}$ direction, and then into the $i \gamma_{5}$ direction [see Eq. (1.2)]. In this situation we again obtain two identical Majorana fermions, except with new phases that are shifted by the same amount, $\theta_{W}^{\prime}=$ $\theta_{W}+\pi / 2$ and $\theta_{m}^{\prime}=\theta_{m}+\pi / 2$. In the continuum limit the explicit topological phase becomes $\theta_{\mathrm{shf}}^{\prime}=\theta+T \theta_{W}^{\prime}=\theta+$ $T\left(\theta_{W}+\pi / 2\right)$. Because the difference $\theta_{W}^{\prime}-\theta_{m}^{\prime}=\theta_{W}-\theta_{m}$ is unchanged, when we substitute the new phases into Eq. (3.26) we see that the effective vacuum angle $\theta_{\text {eff }}$ is unchanged as well.

\section{VACUUM ANGLE AND THE CHIRAL CONDENSATE: COMPLEX IRREP}

Our nonperturbative study in the previous section has implications for the chiral expansion of fermions in a real irrep, and, in particular, for the interplay between the vacuum angle and the $\mathrm{U}(1)$ phase of the fermion mass matrix within the chiral expansion. These will be discussed in Sec. V below. As a preparatory step, in this section we review the role of the vacuum angle in the more familiar case of fermions in a complex irrep. We first consider the chiral condensate in the underlying theory in Sec. IVA, paying special attention to its $U(1)$ phase in the light of the results of the previous section. In Sec. IV B we then discuss how the same features are reproduced in the effective theory, i.e., in chiral perturbation theory.

\section{A. Microscopic theory}

We begin with a continuum derivation. Starting from Eqs. (2.1), (2.2) and (2.3), the left-handed and right-handed fermion condensates are defined by

$$
\begin{aligned}
& \Sigma_{L, i j}=\left\langle\bar{\psi}_{j} P_{L} \psi_{i}\right\rangle=-\frac{1}{V} \frac{\partial \log Z}{\partial \mathcal{M}_{i j}^{*}}, \\
& \Sigma_{R, i j}=\left\langle\bar{\psi}_{j} P_{R} \psi_{i}\right\rangle=-\frac{1}{V} \frac{\partial \log Z}{\partial \mathcal{M}_{j i}},
\end{aligned}
$$

where $V$ is the volume, and $i, j=1, \ldots, N$ are flavor indices. Standard steps using the identity 


$$
\begin{aligned}
& \left(\not D+m\left(\Omega^{\dagger} P_{L}+\Omega P_{R}\right)\right)\left(-\not D+m\left(\Omega P_{L}+\Omega^{\dagger} P_{R}\right)\right) \\
& \quad=-\not D^{2}+m^{2},
\end{aligned}
$$

give rise to the expressions

$$
\begin{gathered}
\Sigma_{L}=-\left(a_{1}-a_{5}\right) \Omega, \\
\Sigma_{R}=-\left(a_{1}+a_{5}\right) \Omega^{\dagger},
\end{gathered}
$$

where

$$
\begin{gathered}
a_{1}=\frac{m}{2 V}\left\langle\operatorname{Tr}\left[\left(-\not D^{2}+m^{2}\right)^{-1}\right]\right\rangle, \\
a_{5}=\frac{m}{2 V}\left\langle\operatorname{Tr}\left[\gamma_{5}\left(-\not D^{2}+m^{2}\right)^{-1}\right]\right\rangle .
\end{gathered}
$$

The Tr symbol indicates a trace over spacetime, color and Dirac indices. ${ }^{10}$ By applying a parity transformation we may express these quantities more explicitly as

$$
\begin{aligned}
& a_{1}=\frac{m}{2 V} \int \mathcal{D} A \tilde{\mu}(A) \cos \left(\theta_{\mathrm{eff}} Q\right) \operatorname{Tr}\left[\left(-\not^{2}+m^{2}\right)^{-1}\right], \\
& a_{5}=-\frac{i m}{2 V} \int \mathcal{D} A \tilde{\mu}(A) \sin \left(\theta_{\mathrm{eff}} Q\right) \operatorname{Tr}\left[\gamma_{5}\left(-D^{2}+m^{2}\right)^{-1}\right] .
\end{aligned}
$$

It follows that $a_{1}$ is real, while $a_{5}$ is imaginary. Both $a_{1}$ and $a_{5}$ are functions of $\theta_{\text {eff }}$, defined in Eq. (2.7). Introducing

$$
z=a_{1}-a_{5}
$$

we arrive at

$$
\begin{aligned}
\Sigma_{L} & =\Sigma_{R}^{\dagger}=-z\left(\theta_{\text {eff }}\right) e^{i \alpha /(N T)} \tilde{\Omega} \\
& =-\left[z\left(\theta_{\text {eff }}\right) e^{-i \theta_{\text {eff }} /(N T)}\right] e^{i \theta /(N T)} \tilde{\Omega} .
\end{aligned}
$$

In the special case $\theta_{\text {eff }}=\theta-\alpha=0, a_{5}$ vanishes while $a_{1}=r$ is real and positive. Hence, in that case, $z=r>0$, and

$$
\Sigma_{L}=-r \Omega=-r e^{i \alpha /(N T)} \tilde{\Omega}=-r e^{i \theta /(N T)} \tilde{\Omega} .
$$

Finally, in the limit $m \rightarrow 0$ we recover the Banks-Casher relation,

$$
r=\frac{\pi}{2} \rho(0),
$$

where $\rho(\lambda)$ is the spectral density of the massless Dirac operator.

\footnotetext{
${ }^{10}$ When the Dirac operator occurs inside the Tr symbol, by convention it carries no flavor indices.
}

Returning to the general case of Eq. (4.10) we see that the orientation of the condensate is determined by the "normalized" mass matrix $\mathcal{M} / m$ and by $\theta_{\text {eff. }}$ In retrospect, this pattern is a consequence of Eq. (4.1), which defines the condensates via derivatives of the partition function with respect to the mass matrix, together with the fact that the partition function itself is invariant under non-Abelian chiral rotations of the mass matrix, and depends on $\theta$ (or $\left.\theta_{\text {shf }}\right)$ and $\alpha$ through their difference $\theta_{\text {eff }}$ only, as we proved rigorously in Sec. III [see, in particular, Eq. (3.26)]. These are the only features of the condensate that we will need in the following.

\section{B. Effective low-energy theory}

We now turn to the effective theory for the NambuGoldstone pions associated with the spontaneous breaking of chiral symmetry. As noted above, at this stage the discussion is restricted to QCD-like theories in which the fermions belong to a complex irrep. The dynamical effective field is

$$
\begin{aligned}
& \Sigma(x)=\Sigma_{0} U(x), \quad U(x)=\exp (i \sqrt{2} \Pi(x) / f), \\
& \Pi(x)=\prod_{a=1}^{N^{2}-1} \Pi_{a}(x) T_{a},
\end{aligned}
$$

where $U(x)$ is the $S U(N)$-valued pion field, and $\Sigma_{0} \in U(1)$ is a constant phase factor. ${ }^{11}$ The leading-order potential is

$$
V=-\frac{f^{2} B}{2} \operatorname{tr}\left(\mathcal{M}^{\dagger} \Sigma+\Sigma^{\dagger} \mathcal{M}\right)
$$

where we recall that $\mathcal{M}=m e^{i \alpha /(N T)} \tilde{\Omega}$, with $\tilde{\Omega} \in S U(N)$. We remind the reader that the product $B m$ is renormalization-group invariant, and depends only on the chiral-limit value of the condensate. ${ }^{12}$

As we have seen in Sec. II A, the partition function of the microscopic theory depends on $\alpha$ and $\theta$ only through their difference $\theta_{\text {eff }}=\theta-\alpha$, and the same must thus be true in the effective theory: the Lagrangian of the effective theory must be a function of $\theta_{\text {eff }}$ only, order by order in the chiral expansion, starting with the tree-level potential $V$. Evidently, $V$ will be a function of only $\theta_{\text {eff }}$ if we set

$$
\Sigma_{0}=e^{i \theta /(N T)} .
$$

In Appendix $\mathrm{E}$ we use the power counting and the symmetries of the effective theory to prove that Eq. (4.15) provides the unique solution to the requirement that the

\footnotetext{
${ }^{11}$ Any constant $S U(N)$-valued part of $\Sigma$ can be absorbed into the pion field. $\Sigma_{0}$ may be regarded as a remnant of the $\eta^{\prime}$ field (see, for instance, Refs. $[9,10]$ ).

${ }^{12}$ In particular, the leading-order chiral Lagrangian is insensitive to the quadratic divergence proportional to $m / a^{2}$ that is present in the bare lattice condensate away from the chiral limit in any fermion formulation.
} 
tree-level potential (4.14) depends on $\alpha$ and $\theta$ only through their difference $\theta_{\text {eff }}$. We also prove that a similar statement applies to the next-to-leading-order Lagrangian.

In the effective theory, the tree-level condensate now takes the form

$$
\Sigma_{L}=\left.\frac{\partial V}{\partial \mathcal{M}^{*}}\right|_{U=U_{0}}=-\frac{f^{2} B}{2} e^{i \theta /(N T)} U_{0},
$$

where $U_{0} \in S U(N)$ is the global minimum of the potential. For this to be consistent with Eq. (4.10), the global minimum $U_{0}$ must be equal to $U_{n}=e^{2 \pi i n / N} \tilde{\Omega}$, for some $0 \leq n<N$, as we will see next. Substituting $U_{n}$ into Eq. (4.14) gives

$$
V\left(U_{n}\right)=-f^{2} B N m \cos \left(\theta_{\text {eff }} /(N T)+2 \pi n / N\right) .
$$

In Appendix $F$ we prove that the global minimum is reached when $\theta_{\text {eff }}+2 \pi n T$ is closest to zero. Denoting the corresponding value of $n$ by $n\left(\theta_{\text {eff }}\right)$, the tree-level condensate is thus

$$
\Sigma_{L}=-\frac{f^{2} B}{2} e^{i\left(\theta /(N T)+2 \pi n\left(\theta_{\text {eff }}\right) / N\right)} \tilde{\Omega} .
$$

This result for $\Sigma_{L}$ is consistent with Eq. (4.10), and thus demonstrates the need to introduce the constant $U(1)$ valued phase $\Sigma_{0}$ into the effective theory. Without $\Sigma_{0}$, the effective theory would have led to a value for $\Sigma_{L}$ in $S U(N)$. This would have been inconsistent, as, for example, can be seen in the case $\theta=\alpha \neq 0$, by comparison with Eq. (4.11).

We comment that in exceptional cases there is a competition between the leading- and next-to-leadingorder potentials [11,12]. In that case the discussion leading to Eq. (4.18) does not apply. But the functional form of Eq. (4.18) remains valid: it must remain true that $\Sigma_{L}$ is oriented in the direction of $e^{i(\theta /(N T)+2 \pi n / N)} \tilde{\Omega}$ for some $n$, where again $n$ depends on $\theta_{\text {eff }}$ only, as can again be seen by comparison with Eq. (4.10).

\section{VACUUM ANGLE AND THE CHIRAL CONDENSATE: REAL IRREP}

In this section we turn to real irreps. In Sec. VA we discuss the condensate, and elaborate on the differences between the complex case (discussed in Sec. IV) and the real case. We deal separately with a standalone theory of Majorana fermions, and with a theory of $2 N$ Majorana fermions that was obtained by reformulating a theory of $N$ Dirac fermions, where the apparent paradox described in the Introduction arises. We then discuss the implications for the chiral effective theory. In Sec. V B we give a diagrammatic proof that, when $\theta_{\text {eff }}$ is held fixed, different orientations of the mass matrix give rise to the same physical observables.

\section{A. The condensate for a real irrep}

We begin with a general theory of $N_{\text {maj }}$ Majorana fermions, where $N_{\text {maj }}$ can be either even or odd. Allowing $N=N_{\text {maj }} / 2$ to be a half integer in Eq. (2.12), the flavor symmetry of the massless theory is $S U\left(N_{\text {maj }}\right)$, which we will assume to be spontaneously broken to $S O\left(N_{\text {maj }}\right)$. We will consider a mass term of the general form

$$
\frac{1}{2} \bar{\Psi}\left(\mathcal{M}^{\dagger} P_{L}+\mathcal{M} P_{R}\right) \Psi
$$

where now

$$
\mathcal{M}=\mathcal{M}^{T}=m \Omega=m e^{2 i \alpha /\left(N_{\text {maj }} T\right)} \tilde{\Omega}, \quad \tilde{\Omega} \in S U\left(N_{\text {maj }}\right),
$$

and we assume $m>0$. Formally, the fermion path integral is a Pfaffian. However, as we have seen in Sec. II C, the phase of this Pfaffian is ambiguous in the continuum. The rigorous solution to this problem is to define the Pfaffian via a lattice regularization. For the mass matrix in Eq. (5.2), this gives rise to the following relations in the continuum limit:

$$
\begin{aligned}
\operatorname{pf}\left(\not D+\mathcal{M}^{\dagger} P_{L}+\mathcal{M} P_{R}\right) & =e^{i \alpha Q} \operatorname{pf}^{N_{\text {maj }}}(\not D+m) \\
& =e^{i \alpha Q_{\operatorname{det}^{N_{\text {maj }}} / 2}(\not D+m) .}
\end{aligned}
$$

The second equality implies that $\operatorname{pf}(\not D+m)$ is strictly positive, as follows from Appendix C. One way to derive Eq. (5.3) is to start from a lattice theory of domain-wall Majorana fermions with $\theta_{W}=0$ and $\theta_{m}=2 \alpha /\left(N_{\text {maj }} T\right)$, and take the continuum limit. Defining $\Sigma_{L}$ and $\Sigma_{R}$ as in Eqs. (4.1a) and (4.1b), but replacing $\psi \rightarrow \Psi$ and $\bar{\psi} \rightarrow \bar{\Psi}$, the rest of the discussion of Sec. IVA carries over. ${ }^{13}$

We next consider the case where $N$ Dirac fermions are traded for $2 N$ Majorana fermions. In the initial Diracfermion lattice formulation we again set $\theta_{W}=0$. As follows from Sec. III, this choice implies that $\theta_{\mathrm{shf}}=\theta$, and thus the angle $\theta$ that multiplies the lattice-discretized topological term in the gauge action is set to the same value as in the target continuum theory. As usual, the $U(1)$ phase of the lattice mass matrix is the same as in the continuum, $\theta_{m}=\alpha /(N T)$.

The key point is that the values of $\theta_{m}$ and $\theta_{W}$ in any equivalent Majorana formulation are constrained by their values in the initial Dirac formulation, and, in particular, by the choice $\theta_{W}=0$ we have initially made. The basic transition to Majorana fermions [using Eq. (2.8) in the Wilson case, or Eq. (3.20) in the domain-wall case] gives rise to a mass term and a (generalized) Wilson term that are both oriented in the direction of the matrix $J_{S}$ of Eq. (1.1). In itself, $J_{S}$ has an axial $U(1)$ phase of $\pi / 2$. As a result, the

\footnotetext{
${ }^{13}$ The definition of parity is somewhat more subtle with Majorana fermions; see Ref. [13].
} 
phases of the mass term and the (generalized) Wilson term both get shifted by $\pi / 2$, becoming $\theta_{m}^{\prime}=\alpha /(N T)+\pi / 2$, and $\theta_{W}^{\prime}=\pi / 2$. In the continuum limit, the new phase of the mass matrix is $\alpha^{\prime}=N T \theta_{m}^{\prime}=\alpha+N T \pi / 2$. The phase $\theta_{W}^{\prime}$ gets traded for an additional vacuum angle, so that the new vacuum angle is $\theta^{\prime}=\theta_{\text {shf }}=\theta+N T \pi / 2$. As expected, both phases were shifted by the same amount, so that the effective vacuum angle, which is their difference, is unchanged, $\theta_{\text {eff }}=\theta-\alpha=\theta^{\prime}-\alpha^{\prime}$.

Alternatively, we may perform an additional (nonanomalous) lattice transformation that brings back the phases to their original values, $\theta_{m}=\alpha /(N T)$ and $\theta_{W}=0$, so that $\theta_{\text {shf }}=\theta$ [for the Wilson case, see Eq. (3.8)]. Once again, $\theta_{\text {eff }}$ is unchanged.

We next turn to the chiral effective theory, focusing on the case $N_{\text {maj }}=2 N$, with the mass matrix $\mathcal{M}$ of Eq. (5.2). The nonlinear field $\Sigma$ is now an element of the coset $S U(2 N) / S O(2 N)$. It is symmetric, $\Sigma^{T}=\Sigma$, and transforms as $\Sigma \rightarrow h \Sigma h^{T}$ under the chiral transformation (2.12), just like $\mathcal{M}$ (when elevated to a spurion). Instead of Eqs. (4.13) and (4.15), which we had in the case of a complex irrep, the coset field for a real irrep is parametrized as

$$
\Sigma(x)=U(x)^{T} \Sigma_{0}=\Sigma_{0} U(x)
$$

where now

$$
\Sigma_{0}=e^{i \tilde{\theta} /(N T)} J
$$

and where $J$ is a real symmetric $S O(2 N)$ matrix. Once again, the phase $\tilde{\theta}$ is to be chosen so that the chiral theory is a function of $\theta_{\text {eff }}$ only. We will discuss examples of this shortly. Equations (5.4) and (5.5) provide a generalization of the results of Ref. [14], where the role of the U(1) phase was not discussed, and of Ref. [15], where the discussion was limited to $\theta=\alpha=0$, and $J=\mathbf{1}_{2 N}$.

For simplicity, in the rest of this section we again set $\tilde{\Omega}=1$ in Eq. (5.2). ${ }^{14}$ Let us consider the construction of the chiral theory in the case we have just discussed, where $N$ Dirac fermions get traded for $2 N$ Majorana fermions. In the initial Dirac formulation we take the mass matrix to be $m e^{i \alpha /(N T)} \mathbf{1}_{N}$, and we allow for an arbitrary vacuum angle $\theta$. After the transition to the Majorana formulation, the mass matrix is $\mathcal{M}=m e^{i \alpha /(N T)} J_{S}$, which is equivalent to a $U(1)$ phase $\alpha^{\prime} /(N T)=\alpha /(N T)+\pi / 2$. Correspondingly, the vacuum angle of the continuum-limit theory becomes $\theta^{\prime}=\theta+N T \pi / 2$. A possible choice for $\Sigma_{0}$ is $e^{i \theta^{\prime} /(N T)} \mathbf{1}_{2 N}$. An alternative, equivalent choice, which involves the same $\mathrm{U}(1)$ phase, is $\Sigma_{0}=e^{i \theta /(N T)} J_{S}$. For the latter choice, the factors of $J_{S}$ cancel out between the mass matrix and the nonlinear field when the latter is expanded in terms of the pion field. Studying the classical solution as we did in Sec. IV B, we similarly find that the expectation value of

\footnotetext{
${ }^{14}$ The generalization to arbitrary $\tilde{\Omega}$ is similar to Sec. IV.
}

the pion field $U(x)$ is a $Z_{2 N}$ element which again depends only on $\theta_{\text {eff }}$.

The situation is similar if we apply an $S U(2 N)$ transformation that rotates the Majorana mass matrix to $\mathcal{M}=$ $m e^{i(\alpha /(N T)+\pi / 2)} \mathbf{1}_{2 N}=m e^{i \alpha^{\prime} /(N T)} \mathbf{1}_{2 N}$ [this corresponds to $J_{S}^{\text {rot }}$ of Eq. (1.2)]. If we choose to apply the same $S U(2 N)$ rotation to $\Sigma_{0}$, it becomes $\Sigma_{0}=e^{i(\theta /(N T)+\pi / 2)} \mathbf{1}_{2 N}=e^{i \theta^{\prime} /(N T)} \mathbf{1}_{2 N}$. Finally, if in the lattice-regularized theory we have applied a further $\mathrm{U}(1)$ axial transformation that simultaneously brings the mass matrix to $\mathcal{M}=m e^{i \alpha /(N T)} \mathbf{1}_{2 N}$, and the (shifted) vacuum angle of the continuum-limit theory back to $\theta_{\text {shf }}=\theta$, then in the chiral theory we can correspondingly set $\Sigma_{0}=e^{i \theta /(N T)} \mathbf{1}_{2 N}$. In all of these examples, the constant mode of the pion field $U(x)$ will be a $Z_{2 N}$ element determined by $\theta_{\text {eff }}$ only.

\section{B. Chiral expansion for a real irrep}

In the case of a complex irrep, studied in Sec. IV, we have demonstrated that the condensate can be expressed as a function of $\theta$ and $\theta_{\text {eff }}$ via Eq. (4.10). We then determined the $\theta$ dependence of the chiral Lagrangian by requiring that the effective theory reproduce this result. When we expand the chiral Lagrangian around the classical solution in terms of the pion field, the expansion is then manifestly a function of $\theta_{\mathrm{eff}}$ only, and not of $\theta$ and $\alpha$ separately. It follows that physical observables, such as the decay constant and the pion mass, depend only on $\theta_{\text {eff }}$ as well.

In the case of a real irrep, we again expect that the chiral expansion for any physical observable will depend on $\alpha$ and $\theta$ only through their difference $\theta_{\text {eff }}$. However, establishing this result is now more subtle. Let us consider two simple examples, both of which can be parametrized as $\mathcal{M}=m J$, $\Sigma_{0}=J$, for the same $J$. The two cases are then defined by taking $J=J_{S}$, for which $\alpha /(N T)=\theta /(N T)=\pi / 2$, or $J=\mathbf{1}_{2 N}$, for which $\alpha=\theta=0$. Notice that $\theta_{\text {eff }}=0$ in both cases. Now, using Eq. (5.4), and noting that in both cases $J^{2}=\mathbf{1}_{2 N}$, it is easy to see that $J$ drops out of the product $\Sigma^{\dagger}(x) \mathcal{M}$. However, unlike in the case of a complex irrep, this does not immediately imply that the perturbative expansion is independent of the choice of $J$. The reason is the constraints imposed on the pion field: this field is Hermitian, traceless, and satisfies

$$
\pi=J \pi^{T} J
$$

Thus, even though $J$ drops out of the tree-level Lagrangian, the pion field still depends on it, through the above constraint, and the pion propagator $[14,15]$

$$
\begin{aligned}
& \left\langle\pi_{i j}(x) \pi_{k \ell}(y)\right\rangle \\
& =\int \frac{d^{4} p}{(2 \pi)^{4}} \frac{e^{i p(x-y)}}{p^{2}+M^{2}}\left(\frac{1}{2}\left(\delta_{i \ell} \delta_{j k}+J_{i k} J_{j \ell}\right)-\frac{1}{2 N} \delta_{i j} \delta_{k \ell}\right),
\end{aligned}
$$

depends on the choice of $J$ explicitly. 
Let us consider the case $N=1$. For $J=J_{S}$, and choosing a basis where $J_{S}=\sigma_{3}$, the constraints translate into $\pi_{11}=\pi_{11}^{*}=-\pi_{22}$, and $\pi_{12}=-\pi_{12}^{*}=-\pi_{21}$. For $J=\mathbf{1}_{2}$, the diagonal elements remain the same as before, whereas for the off-diagonal elements we have $\pi_{12}=\pi_{12}^{*}=\pi_{21}$. Stated differently, for $J=\sigma_{3}$ the expansion of the pion field is $\pi=\pi_{3} \sigma_{3}+\pi_{2} \sigma_{2}$, whereas for $J=\mathbf{1}_{2}$ it is $\pi=\pi_{3} \sigma_{3}+\pi_{1} \sigma_{1}$. The tensor structure of the nonvanishing propagators is

$$
\begin{aligned}
\left\langle\pi_{11}(x) \pi_{11}(y)\right\rangle: & \frac{1}{2}\left(\delta_{11} \delta_{11}+J_{11} J_{11}\right)-\frac{1}{2} \delta_{11} \delta_{11}=\frac{1}{2}, \\
J=\sigma_{3}, \mathbf{1}_{2}, & \\
\left\langle\pi_{12}(x) \pi_{12}(y)\right\rangle: & \frac{1}{2}\left(\delta_{12} \delta_{12}+J_{11} J_{22}\right)-\frac{1}{2} \delta_{12} \delta_{12} \\
& = \begin{cases}-\frac{1}{2}, & J=\sigma_{3}, \\
\frac{1}{2}, & J=\mathbf{1}_{2} .\end{cases}
\end{aligned}
$$

Using a hat to distinguish the pion field for the case $J=\sigma_{3}$, we see that it will transform into the pion field of the $J=\mathbf{1}_{2}$ case if we substitute

$$
\hat{\pi}_{11}=\pi_{11}, \quad \hat{\pi}_{12}=i \pi_{12},
$$

which corresponds to the replacement of $\sigma_{1}$ by $\sigma_{2}$ in the expansion of the pion field. Of course, nonperturbatively, the redefinition (5.9) is not allowed, but in (chiral) perturbation theory the only question is whether it leads to the same order-by-order diagrammatic expansion for any correlation function with a prescribed set of external pion legs. We will now prove that

$$
\begin{aligned}
& \left\langle\hat{\pi}_{11}^{(1)}\left(x_{1}\right) \ldots \hat{\pi}_{11}^{(m)}\left(x_{m}\right) \hat{\pi}_{12}^{(1)}\left(y_{1}\right) \ldots \hat{\pi}_{12}^{(n)}\left(y_{n}\right)\right\rangle \\
& \quad=i^{n}\left\langle\pi_{11}^{(1)}\left(x_{1}\right) \ldots \pi_{11}^{(m)}\left(x_{m}\right) \pi_{12}^{(1)}\left(y_{1}\right) \ldots \pi_{12}^{(n)}\left(y_{n}\right)\right\rangle,
\end{aligned}
$$

to all orders in chiral perturbation theory, for any $m$ and $n$.

A vertex with $k \pi_{12}$ lines attached to it also changes by a factor $i^{k}$ after the field redefinition (note that $k$ is always even, so that taking $i$ or $-i$ does not matter). Also, for any diagram, the number of $\pi_{12}$ external lines $n$, the number of $\pi_{12}$ propagators $p$ and the number $v_{k}$ of vertices with $k \pi_{12}$ lines attached to it are related by

$$
2 p=n+\sum_{k} k v_{k} .
$$

It follows from this relation that, for all diagrams, the field redefinition (5.9) indeed leads to the factor $i^{n}$ in Eq. (5.10), thus proving this result. Each $\pi_{12}$ propagator flips its sign, and $p$ such propagators thus lead to a factor $(-1)^{p}=i^{2 p}$. In addition, the diagram changes by a factor $i \sum_{k}{ }^{k} v_{k}$ because of the $v_{k}$ vertices with $k \pi_{12}$ lines, and thus the diagram changes by a total factor $i^{2 p+\sum_{k} k v_{k}}=i^{n}$, using Eq. (5.11).
Here we also used that all terms in the exponent are even (and, thus, $n$ is even as well).

Next, we discuss the general case of $N$ Dirac fermions in a real irrep, comparing the cases $J=J_{S}$, with $J_{S}$ in Eq. (1.1), and $J=\mathbf{1}_{2 N}$. The matrix $J_{S}$ can now be brought into a form in which $\sigma_{3}$ appears $N$ times along the diagonal. The constraints on the pion field are now, in this basis,

$$
\begin{aligned}
\pi_{N N} & =-\sum_{i=1}^{N-1} \pi_{i i}, \\
\pi_{i j} & =(-1)^{i+j} \pi_{j i} .
\end{aligned}
$$

In addition, $\pi_{i i}$ is real for all $i$, and $\pi_{i j}=\pi_{j i}^{*}$ for all $i \neq j$. A minus sign in the pion propagator $\left\langle\pi_{i j}(x) \pi_{i j}(y)\right\rangle$, cf. Eq. (5.8), occurs when $i$ is even and $j$ is odd, or the other way around, because $J_{i i} J_{j j}=-1$ only in this situation. Since minus signs in a field redefinition like Eq. (5.9) do not affect our arguments, we can choose

$$
\hat{\pi}_{i j}=i^{i+j} \pi_{i j}
$$

Now let us consider a diagram with $p_{i j} \hat{\pi}_{i j}$ propagators, $n_{i j}$ $\hat{\pi}_{i j}$ external lines, and $v_{k, i j}$ vertices with $k_{i j} \hat{\pi}_{i j}$ lines attached to it. Note that because of Eq. (5.12) we can always take $i \leq j$ (and $i \neq N$ if $i=j$, but this is not important). We have that

$$
2 p_{i j}=n_{i j}+\sum_{k_{i j}} k_{i j} v_{k, i j}
$$

This relation implies that a correlation function with $n_{i j}$ external $\hat{\pi}_{i j}$ lines equals $i^{-(i+j) n_{i j}}$ times the correlation function in terms of the unhatted meson field $\pi_{i j}$, using that $i^{-2 p_{i j}}=i^{2 p_{i j}}$, and Eq. (5.14). The full correlation function changes by the product

$$
\prod_{i j} i^{-(i+j) n_{i j}}=i^{-\sum_{i j}(i+j) n_{i j}},
$$

where the product and sum are over all pairs $i j$ present in the correlation function. The sum in the exponent on the right-hand side of Eq. (5.15) always has to be even, because every index has to appear an even number of times in the correlation function for it to not vanish. This means we can drop the minus sign in this exponent, and we thus find the desired result.

Note that, unlike in the $N=1$ example, we do not always have that $n_{i j}$ is even. A simple counter example is the correlation function $\left\langle\pi_{12} \pi_{23} \pi_{34} \pi_{41}\right\rangle$, which does not vanish, but has $n_{12}=n_{23}=n_{34}=n_{14}=1$. However, clearly, $(1+2) n_{12}+(2+3) n_{23}+(3+4) n_{34}+(1+4) n_{14}=20$ is even.

A similar type of argument was used in Ref. [16] to show the equivalence of "standard" quenched chiral perturbation 
theory [17] with "nonperturbatively correct" quenched chiral perturbation theory.

\section{CONCLUSION}

In QCD-like theories it is well known that physical observables depend only on the effective vacuum angle $\theta_{\text {eff }}$, which is the difference between the explicit angle $\theta$ multiplying the topological term in the gauge-field action, and the (properly normalized) $U(1)_{A}$ angle $\alpha$ of the fermion mass matrix.

When $N$ Dirac fermions belong to a real irrep of the gauge group, the theory can be reformulated in terms of $2 \mathrm{~N}$ Majorana fermions. The integration over a Majorana field yields a functional Pfaffian. As we discussed in the Introduction, the phase of this Pfaffian appears to lead to a paradox: in certain cases, $\theta_{\text {eff }}$ changes by an odd multiple of $\pi$ relative to its value in the initial Dirac theory. Tracing the origin of this phenomenon we showed that, in the continuum, the phase of the functional Pfaffian is in fact inherently ambiguous, as it depends on the choice of basis for the Majorana field. A partial solution is that, in the case of $2 N$ Majorana fermions, one can fix the ambiguity by appealing to the corresponding theory of $N$ Dirac fermions in such a way that the apparent paradox is avoided.

A nonperturbative lattice definition of Majorana fermions is free of the phase ambiguity: on any finite-volume lattice, the (real-irrep) Dirac operator becomes a finitesize matrix, and, moreover, the lattice automatically selects the coordinate basis to define the Dirac operator, and, hence, its Pfaffian. We reviewed the work of Ref. [1] who argued long ago that, if the Wilson term in the Wilson lattice action for Dirac fermions is rotated by a phase, that phase induces a topological term in the continuum limit. We observed that there is a subtlety with this argument associated with renormalization, which leads to a conjecture (first made in Ref. [18]) on how to complete the argument of Ref. [1], described in Appendix D. We generalized this result to domain-wall fermions, where this subtlety does not arise, as well as to the case of Majorana fermions. This allowed us to unambiguously determine the effective vacuum angle, finding consistent results between the Dirac and Majorana formulations in all cases.

As an application, we discussed how chiral perturbation theory reproduces the correct dependence on the explicit $(\theta)$ and effective $\left(\theta_{\text {eff }}\right)$ vacuum angles. This behavior has been long known (even if maybe not widely known) for the effective theory for a gauge theory with Dirac fermions, but, to our knowledge, this is the first detailed study of this issue for the effective theory for a gauge theory with Majorana fermions. As such, our results fill in a lacuna in the discussion of Ref. [14], and resolve a question that was left open in Ref. [15]. In particular, we considered the chiral expansion for $2 N$ Majorana fermions in two cases that share $\theta_{\text {eff }}=0$, while the mass matrix is proportional to $J_{S}$ in one case, and to $\mathbf{1}_{2 N}$ in the other, giving a diagrammatic proof that all physical observables are equal in the two cases, as required by the common value of $\theta_{\text {eff }}$.

\section{ACKNOWLEDGMENTS}

We like to thank Steve Sharpe for useful discussions. We also like to thank Jan Smit for comments and discussion on the first version of this paper, which led to the addition of two new appendices. The work of M. G. is supported by the U.S. Department of Energy, Office of Science, Office of High Energy Physics, under Award No. DE-FG0392ER40711. Y.S. is supported by the Israel Science Foundation under Grant No. 491/17.

\section{APPENDIX A: SPECTRAL REPRESENTATION OF THE DIRAC OPERATOR}

Consider the one-flavor Dirac operator for a general complex mass,

$$
D=\not D+m e^{i \gamma_{5} \tilde{\alpha}}
$$

where

$$
\not D=-\not^{\dagger}=\left(\begin{array}{cc}
0 & \bar{\sigma}_{\mu} D_{\mu} \\
\sigma_{\mu} D_{\mu} & 0
\end{array}\right),
$$

with $\sigma_{\mu}=\left(\mathbf{1}_{2}, i \vec{\sigma}\right)$ and $\bar{\sigma}_{\mu}=\left(\mathbf{1}_{2},-i \vec{\sigma}\right)$. Let us derive the spectral representation of $\operatorname{det}(D)$ (see, for example, Ref. [19]). For a zero mode, depending on its chirality, the eigenvalue is simply $m e^{ \pm i \tilde{\alpha}} \equiv m_{1} \pm i m_{2}$. Turning to the nonzero modes we start with the right-handed spectrum of the second-order operator,

$$
-D^{2} \psi_{R}=-\left(\bar{\sigma}_{\mu} D_{\mu}\right)\left(\sigma_{\nu} D_{\nu}\right) \psi_{R}=\lambda^{2} \psi_{R},
$$

where we take $\lambda$ real and positive. We consider the following ansatz for an eigenvector of $D$ :

$$
\begin{array}{r}
\left(\begin{array}{cc}
m e^{i \tilde{\alpha}} & \bar{\sigma}_{\mu} D_{\mu} \\
\sigma_{\mu} D_{\mu} & m e^{-i \tilde{\alpha}}
\end{array}\right)\left(\begin{array}{c}
\psi_{R} \\
c \sigma_{\nu} D_{\nu} \psi_{R}
\end{array}\right) \\
=\left(\begin{array}{c}
\left(-c \lambda^{2}+m e^{i \tilde{\alpha}}\right) \psi_{R} \\
\left(1+c m e^{-i \tilde{\alpha}}\right) \sigma_{\mu} D_{\mu} \psi_{R}
\end{array}\right),
\end{array}
$$

where the components of each column vector correspond to the two chiralities. Requiring that the column vector on the left-hand side is an eigenvector gives rise to a quadratic equation for $c$, with the two solutions

$$
c_{ \pm}=\frac{i m_{2}}{\lambda^{2}} \pm \frac{i}{\lambda} \sqrt{1+\frac{m_{2}^{2}}{\lambda^{2}}}
$$

We denote the resulting eigenvectors by $\chi_{ \pm}$. The corresponding eigenvalues are 


$$
\lambda_{ \pm}=m_{1} \mp i \sqrt{\lambda^{2}+m_{2}^{2}} .
$$

The product of the two eigenvalues is $\lambda_{+} \lambda_{-}=\lambda^{2}+m^{2}$. Remembering that there are $T$ zero modes per instanton, it follows that the determinant of the one-flavor Dirac operator (A1) is

$$
\operatorname{det}\left(\not D+m e^{i \tilde{\alpha} \gamma_{5}}\right)=\left(m e^{i \tilde{\alpha}}\right)^{T Q} \prod_{\lambda>0}\left(\lambda^{2}+m^{2}\right) .
$$

The first factor on the right-hand side is the contribution of the zero modes, where $Q$ is the topological charge of the (multi)instanton background field. The second factor gives the contribution of the nonzero modes in terms of the eigenvalues of the second-order operator. For the $N$-flavor case, substituting $\tilde{\alpha}=\alpha /(N T)$ gives rise to Eq. (2.4).

\section{APPENDIX B: MAJORANA BASES}

Let us consider for definiteness the case of a single Dirac fermion. If we follow the basis transformations that lead to Eq. (3.7) and then to Eq. (3.8) we arrive at the following relations:

$$
\begin{aligned}
& \Psi_{1}=\left(\Psi_{1}^{\prime}-i \gamma_{5} \Psi_{2}^{\prime}\right) / \sqrt{2} \\
& \Psi_{2}=\left(\Psi_{1}^{\prime}+i \gamma_{5} \Psi_{2}^{\prime}\right) / \sqrt{2},
\end{aligned}
$$

where the new Majorana fields $\Psi_{1,2}^{\prime}$ correspond to the diagonal form of the Dirac operator in Eq. (3.8). The original Dirac field can be expressed as

$$
\begin{aligned}
& \psi=P_{L} \Psi_{1}+P_{R} \Psi_{2}=\left(\Psi_{1}^{\prime}+i \Psi_{2}^{\prime}\right) / \sqrt{2}, \\
& \bar{\psi}=\left(\bar{\Psi}_{1}^{\prime}-i \bar{\Psi}_{2}^{\prime}\right) / \sqrt{2},
\end{aligned}
$$

where we have used Eq. (B1). Equation (B2) is reminiscent of the original notion of Majorana fields in Minkowski space as the real and imaginary parts of a Dirac field, for a real irrep. As we explained in Sec. II B, in this paper we prefer the basis (2.8), because it respects the natural correspondence between Weyl and Majorana fields.

We stress that the existence of the above basis, in which the Majorana mass matrix is diagonal [see Eq. (3.8)], does not resolve the puzzle we discussed in the Introduction; that puzzle must find its resolution when the basis (2.8) is used, and indeed it does, as we showed in Sec. III.

\section{APPENDIX C: POSITIVITY OF THE DOMAIN-WALL PFAFFIAN FOR POSITIVE MASS}

In this Appendix we prove that the Pfaffian of a single domain-wall Majorana fermion is positive for a positive bare mass, i.e., in the absence of chiral phases.
We first consider the Dirac case. We write the fermion action as $S=\bar{\psi} D_{D W}\left(m_{0}\right) \psi$, where the explicit form of $D_{D W}\left(m_{0}\right)$ may be read off from Eq. (3.9). It was proved in Ref. [7] that the partition function of a domain-wall fermion, $\operatorname{det}\left(D_{D W}\right)$, is strictly positive when $m_{0}>0$. Turning to the case with one Majorana fermion, and introducing the antisymmetric $\mathcal{A}_{D W}\left(m_{0}\right)=R_{5} C S D_{D W}(U)$, our task is to prove that $\operatorname{pf}\left(\mathcal{A}_{D W}\left(m_{0}\right)\right)$ is strictly positive as well.

The argument uses elementary calculus. We consider a finite lattice, so that configuration space is compact. Since $\operatorname{det}\left(D_{D W}(U)\right)>0$ for any gauge-field configuration $U$, it follows that there exists $\mu>0$ such that $\operatorname{det}\left(D_{D W}(U)\right) \geq \mu^{2}$, for all $U$. If this were not true, we could find an infinite sequence of configurations $U_{i}$, such that $\lim \operatorname{det}\left(D_{D W}\left(U_{i}\right)\right) \rightarrow 0$. Because of compactness, that sequence would have a convergent subsequence $U_{i}^{\prime} \rightarrow U_{\text {lim }}$, where $U_{\text {lim }}$ is a gauge configuration too. It would then follow that $\operatorname{det}\left(D_{D W}\left(U_{\lim }\right)\right)=0$, contrary to the result that $\operatorname{det}\left(D_{D W}(U)\right)>0$ for all configurations.

Next, we have $\operatorname{pf}^{2}\left(\mathcal{A}_{D W}(U)\right)=\operatorname{det}\left(D_{D W}(U)\right)$. Therefore, either $\operatorname{pf}\left(\mathcal{A}_{D W}(U)\right) \geq \mu$ or $\operatorname{pf}\left(\mathcal{A}_{D W}(U)\right) \leq-\mu$. Moreover, $\operatorname{pf}\left(\mathcal{A}_{D W}(\mathbf{1})\right)$ is positive for the free case $U=\mathbf{1}$.

It follows that $\operatorname{pf}\left(\mathcal{A}_{D W}(U)\right) \geq \mu$ for all configurations $U$. If this were not true, there would be a configuration $U_{0}$ for which $\operatorname{pf}\left(\mathcal{A}_{D W}\left(U_{0}\right)\right)$ is negative. Now choose a smooth path $U(t)$ such that $U(0)=\mathbf{1}$ and $U(1)=U_{0}$. Along this path, $\operatorname{pf}\left(\mathcal{A}_{D W}(U(t))\right.$ must change continuously from positive to negative, and thus go through zero. But, this is impossible, because we have seen that $\left|\operatorname{pf}\left(\mathcal{A}_{D W}(U)\right)\right| \geq \mu$.

\section{APPENDIX D: DISCUSSION OF THE RESULT OF REF. [1]}

As was shown long ago in Ref. [20] in the theory with $\theta_{W}=\theta_{m}=0$, the bare mass $m_{0}$ in Eq. (3.1) renormalizes additively. This is related to the fact that there is no symmetry distinguishing between the Wilson term $W$ and the single-site term proportional to $m_{0}$. This fact was not considered in Ref. [1]. The proof of the observation quoted in Eq. (3.4) was given for a theory with Wilson fermions in the semiclassical limit, i.e., in the presence of a smooth background gauge field. The additive renormalization thus does not arise, as it is caused by quantum fluctuations of the gauge field. In this appendix we describe a conjecture on the interplay of the observation of Ref. [1] and renormalization. Our discussion here largely overlaps with Ref. [18].

With quantum effects thus "mixing" the Wilson and single-site mass terms in Eq. (3.1), the question arises whether two angles $\theta_{W}$ and $\theta_{m}$ can be unambiguously introduced, and, if so, how this should be done. Here, we will discuss the issue, and formulate a natural conjecture answering this question. A rigorous proof of our conjecture is outside the scope of this paper. 
First, consider a lattice gauge theory with Wilson fermions without any $\theta$ angles which leads to massless fermions in the continuum limit. It follows from Ref. [20] that in order to construct such a theory, the bare mass $m_{0}$ needs to be tuned to a critical value $m_{c}$ that depends on the bare coupling, i.e., the massless theory is obtained from a lattice theory with fermion operator $D_{K}+W+m_{c}$. ${ }^{15}$ Using an axial rotation of the form (3.3), we can introduce an angle $\theta_{W}$ in this theory, turning the fermion operator into $D_{K}+e^{i \gamma_{5} \theta_{W}}\left(W+m_{c}\right)$. Of course, in the massless theory, this angle has no physical consequence, consistent with what one expects in a massless continuum theory. This construction does imply that if one starts with a theory with Wilson term $e^{i \gamma_{5} \theta_{W}} W$, the critical mass $m_{c}$ introduced to obtain a massless continuum limit also needs to be multiplied by $e^{i \gamma_{5} \theta_{W}}$.

Next, we may introduce a physical mass, which here we will take to be the axial-Ward-identity (AWI) mass $m_{\mathrm{AWI}}$, by choosing

$$
m_{0}=m_{c}+Z m_{\mathrm{AWI}}
$$

where we also introduced the multiplicative renormalization constant $Z$ relating the bare subtracted lattice mass $m_{0}-m_{c}$ and the renormalized mass $m_{\mathrm{AWI}}$ [20,22]. We may now introduce another angle $\theta_{m}$ by considering the operator

$D_{W}\left(\theta_{W}, \theta_{m}\right)=D_{K}+e^{i \gamma_{5} \theta_{W}}\left(W+m_{c}\right)+e^{i \gamma_{5} \theta_{m}} Z m_{\mathrm{AWI}}$.

We recall that $m_{c}$ has already been determined for $m_{\mathrm{AWI}}=0$, and that it is independent of both $\theta_{W}$ and $\theta_{m}$. The question arises how the $Z$ factor depends on these angles. In order to address this question, we first apply an axial rotation (3.3) with $\eta=-\theta_{W} / 2$ to remove the phase of the Wilson term, arriving at

$$
D_{W}^{\prime}\left(\theta_{W}, \theta_{m}\right)=D_{K}+\left(W+m_{c}\right)+e^{i \gamma_{5}\left(\theta_{m}-\theta_{W}\right)} Z m_{\mathrm{AWI}} .
$$

At tree level, the Wilson-Dirac operator $D_{W}^{\prime}$ is now a function of the difference $\theta_{m}-\theta_{W}$, and, by necessity, the same applies to the $Z$ factor, order by order in perturbation theory. The last step is to undo the axial rotation, going from $D_{W}^{\prime}$ back to $D_{W}$. Assuming that our renormalization condition transforms covariantly under axial rotations, ${ }^{16}$ the same $Z$ factor that we have determined for $D_{W}^{\prime}$ will continue to satisfy the corresponding renormalization

\footnotetext{
${ }^{15}$ The precise definition of $m_{c}$ is subject to ambiguities of order $a^{2}$ [21]. However, we will assume that we are in the scaling region, where these ambiguities can be ignored.

${ }^{16}$ This includes as a special case any renormalization condition which is invariant under axial rotations of the fields.
}

condition for $D_{W}$. It follows that, in Eq. (D2), and for general values of $\theta_{W}$ and $\theta_{m}, Z$ is a function of the difference $\theta_{m}-\theta_{W}$ only. We comment that the universal, logarithmic part of the $Z$ factor is actually independent of $\theta_{W}$ and $\theta_{m}$. However, this $Z$ factor also has a finite part, and that part will in general depend on $\theta_{W}$ and $\theta_{m}$, but, as we have just argued, only through their difference.

Our conjecture is that in the fully dynamical theory Eq. (3.4) holds, with the fermion operator as defined in Eq. (D2). We observe that this conjecture is natural, in the sense that, in the continuum limit, the mass $m_{\mathrm{AWI}}$ is the fermion mass $m$ to be used in Secs. IV and V.

\section{APPENDIX E: $\theta$ DEPENDENCE OF THE CHIRAL THEORY}

As in Sec. IV B we consider here a gauge theory with $N$ Dirac fermions in some complex irrep. For $\theta=0$, the chiral Lagrangian is constructed using the nonlinear field $U(x) \in S U(N)$; see Eq. (4.13). We will prove that, at both leading order (LO) and next-to-leading order (NLO), the chiral Lagrangian for $\theta \neq 0$ is obtained via the replacement $U(x) \rightarrow \Sigma(x)$, where $\Sigma(x)=U(x) \Sigma_{0}$ [see Eq. (4.13)], and where $\Sigma_{0}$ is given by Eq. (4.15). ${ }^{17}$ As before, $\mathcal{M}$ is given in Eq. (2.3).

We start at tree level. The requirement that the Lagrangian of the chiral theory depend on $\theta$ and $\alpha$ only through their difference $\theta_{\text {eff }}$ is satisfied if the potential admits the form

$$
V=-\frac{f^{2} B}{2} \operatorname{tr}\left(e^{i f\left(\theta_{\text {eff }}\right)} \mathcal{M}^{\dagger} \Sigma+\text { H.c. }\right) .
$$

This amounts to multiplying $\mathcal{M}^{\dagger} \Sigma$ in Eq. (4.14) by the phase factor $e^{i f\left(\theta_{\text {eff }}\right)}$, where $f\left(\theta_{\text {eff }}\right)$ is a priori an arbitrary (real) function of its argument.

We first invoke the chiral power counting, which implies that the tree-level Lagrangian should be linear in $\mathcal{M}$ or $\mathcal{M}^{\dagger}$. This dependence is already explicit in Eq. (E1), and so

$$
f\left(\theta_{\text {eff }}\right)=f(\theta-\alpha)=f(\theta-\operatorname{Im} \log \operatorname{det} \mathcal{M}),
$$

must in fact be independent of $\mathcal{M}$. This allows us to set $f\left(\theta_{\text {eff }}\right)=c$ in Eq. (E1), where $c$ is some constant.

Next we consider the special case where $\mathcal{M}=m \mathbf{1}_{N}$, with $m>0$, and $\theta=0$. Now $\Sigma=U$ and the tree-level Lagrangian must be invariant under the (internal) parity transformation $U(x) \rightarrow U^{\dagger}(x)$. This invariance is respected only for $e^{i c}= \pm 1$, which completes the argument. ${ }^{18}$

The reasoning at NLO is similar. A priori, $\mathcal{M}^{\dagger} \Sigma$ can again be multiplied by a phase factor $e^{i f\left(\theta_{\text {eff }}\right)}$, where $f\left(\theta_{\text {eff }}\right)$ is a new arbitrary real function for each occurrence of $\mathcal{M}^{\dagger} \Sigma$. But, as before, the power counting restricts every

\footnotetext{
${ }^{17} \mathrm{We}$ conjecture that a similar statement applies to all orders in the chiral expansion.

${ }^{18}$ The choice $e^{i c}=+1$ is conventional.
} 
such $f\left(\theta_{\text {eff }}\right)$ to a constant. In the last step we consider the most general constant phase factors consistent with parity invariance of the $\mathcal{M}=m \mathbf{1}_{N}, \theta=0$ theory, finding that this does not give rise to any new operators not already present in the standard NLO chiral Lagrangian.

\section{APPENDIX F: PROOF OF EQ. (4.18)}

Let us prove, algebraically, that the global minimum of the tree-level potential (4.14) is given by $\Sigma_{L}$ of Eq. (4.18), with $n=n\left(\theta_{\text {eff }}\right)$ as described in Sec. IV B.

We begin by writing the $S U(N)$ matrix $U$ of Eq. (4.13) as $U=\tilde{\Omega} \tilde{U}$, so that the potential becomes

$$
V=-\frac{f^{2} B m}{2} \operatorname{tr}\left(e^{i \theta_{\text {eff }} /(N T)} \tilde{U}+\text { H.c. }\right) .
$$

We may assume without loss of generality that $\tilde{U}$ is diagonal,

$$
\tilde{U}=\operatorname{diag}\left(e^{i \phi_{1}}, e^{i \phi_{2}}, \ldots, e^{i \phi_{N-1}}, e^{i \phi_{N}}\right),
$$

where $\phi_{1}, \ldots, \phi_{N-1}$ are the independent real variables, and

$$
\phi_{N}=2 \pi n-\left(\phi_{1}+\cdots+\phi_{N-1}\right),
$$

where $n$ is an arbitrary integer. Introducing the shorthand $\tilde{\theta}=\theta_{\text {eff }} /(N T)$ we need to find the global maximum of

$$
\mathcal{V}=\frac{1}{2} \operatorname{tr}\left(e^{i \tilde{\theta}} \tilde{U}+\text { H.c. }\right)=\sum_{k=1}^{N} \cos \left(\tilde{\theta}+\phi_{k}\right) .
$$

The saddle-point conditions are

$$
\sin \left(\tilde{\theta}+\phi_{k}\right)=\sin \left(\tilde{\theta}+\phi_{N}\right), \quad k=1,2, \ldots, N-1 .
$$

First consider a solution with all phases equal. Equation (F3) then implies that $\phi_{k}=2 \pi n / N, k \in\{1, \ldots, N\}$, for some $n$, and $\mathcal{V}=N \cos (\tilde{\theta}+2 \pi n / N)$. The global maximum over this set of solutions is obtained for $n=n\left(\theta_{\text {eff }}\right)$, defined as before as the value of $n$ for which $\tilde{\theta}+2 \pi n / N$ is closest to zero. The value of this maximum is

$$
\mathcal{V}_{\max }=N \cos \left(\theta_{\text {eff }} /(N T)+2 \pi n\left(\theta_{\text {eff }}\right) / N\right),
$$

which reproduces Eq. (4.17).
It remains to prove that this solution is in fact the global maximum of $\mathcal{V}$ over the entire set of saddle points. What complicates matters is that Eq. (F5) can be satisfied by $\phi_{k}=\phi_{N}$, or by $\phi_{k}=\pi-2 \tilde{\theta}-\phi_{N}$. In the former case we have $\cos \left(\tilde{\theta}+\phi_{k}\right)=\cos \left(\tilde{\theta}+\phi_{N}\right)$, whereas in the latter case we have $\cos \left(\tilde{\theta}+\phi_{k}\right)=-\cos \left(\tilde{\theta}+\phi_{N}\right)$, so that $\cos \left(\tilde{\theta}+\phi_{k}\right)+\cos \left(\tilde{\theta}+\phi_{N}\right)=0$.

Let us denote by $\mathcal{V}_{\max }^{(1)}$ the maximal value of $\mathcal{V}$ when $\phi_{1}=\pi-2 \tilde{\theta}-\phi_{N}$, while the remaining $N-2$ independent phases are equal to $\phi_{N}$. It follows immediately from the above discussion that in this case $\mathcal{V}=\sum_{k=3}^{N} \cos \left(\tilde{\theta}+\phi_{k}\right)=$ $(N-2) \cos \left(\tilde{\theta}+\phi_{N}\right)$, leading to the upper bound

$$
\mathcal{V}_{\max }^{(1)} \leq N-2 .
$$

Similarly, if exactly two independent phases are equal to $\pi-2 \tilde{\theta}-\phi_{N}$, then the corresponding maximal value is bounded by $\mathcal{V}_{\max }^{(2)} \leq N-4$, and so on.

We also need a lower bound on the maximum in Eq. (F6). The maximum value the angle $\tilde{\theta}+2 \pi n\left(\theta_{\text {eff }}\right) / N$ can take is equal to $\pi / N$. Since $\sin (x) \leq x$, this implies

$$
\cos \left(\theta_{\text {eff }} /(N T)+2 \pi n\left(\theta_{\text {eff }}\right) / N\right) \geq \sqrt{1-(\pi / N)^{2}} .
$$

It follows that $\mathcal{V}_{\max }$ of Eq. (F6) is larger than $\mathcal{V}_{\max }^{(1)}$ if

$$
N \sqrt{1-(\pi / N)^{2}} \geq N-2
$$

which is true for $N \geq 4$.

It remains to check explicitly the cases $N=2,3$. For $N=2$, choosing $\phi_{1}=\pi-2 \tilde{\theta}-\phi_{2}$ gives $\mathcal{V}=0$, which is smaller than the maximum in Eq. (F6). ${ }^{19}$

For $N=3$, if we choose $\phi_{1}=\pi-2 \tilde{\theta}-\phi_{3}$ and $\phi_{2}=\phi_{3}$ then $\mathcal{V}_{\max }^{(1)} \leq 1$ according to the upper bound (F7). By contrast, for the solution with $\phi_{1}=\phi_{2}=\phi_{3}$, the maximum value of the angle $\tilde{\theta}+2 \pi n\left(\theta_{\text {eff }}\right) / N$ is now $\pi / 3$; hence, $N \cos \left(\theta_{\text {eff }} /(N T)+2 \pi n\left(\theta_{\text {eff }}\right) / N\right)$ is bounded from below by $3 / 2$, making $\mathcal{V}_{\max }$ again the true global maximum.

\footnotetext{
${ }^{19}$ An exception is the case $\tilde{\theta}=\pi / 2$, for which Eq. (F6) vanishes, too. In fact, the tree-level potential is identically zero in this case [11]. For $\tilde{\theta}$ close to $\pi / 2$ there is competition between LO and NLO, and the above discussion does not apply.
} 
[1] E. Seiler and I. O. Stamatescu, Lattice Fermions and $\theta$ vacua, Phys. Rev. D 25, 2177 (1982); Phys. Rev. D 26, 534 (E) (1982).

[2] T. DeGrand, Y. Liu, E. T. Neil, Y. Shamir, and B. Svetitsky, Spectroscopy of SU(4) gauge theory with two flavors of sextet fermions, Phys. Rev. D 91, 114502 (2015).

[3] H. S. Sharatchandra, H. J. Thun, and P. Weisz, Susskind Fermions on a Euclidean lattice, Nucl. Phys. B192, 205 (1981).

[4] C. van den Doel and J. Smit, Dynamical symmetry breaking in two flavor $\mathrm{SU}(N)$ and $\mathrm{SO}(N)$ lattice gauge theories, Nucl. Phys. B228, 122 (1983).

[5] D. B. Kaplan, A method for simulating chiral fermions on the lattice, Phys. Lett. B 288, 342 (1992).

[6] Y. Shamir, Anomalies and chiral defects fermions, Nucl. Phys. B417, 167 (1994).

[7] V. Furman and Y. Shamir, Axial symmetries in lattice QCD with Kaplan fermions, Nucl. Phys. B439, 54 (1995).

[8] D. B. Kaplan and M. Schmaltz, Supersymmetric Yang-Mills theories from domain wall fermions, Chin. J. Phys. 38, 543 (2000).

[9] E. Witten, Large N Chiral dynamics, Ann. Phys. (N.Y.) 128, 363 (1980).

[10] P. Di Vecchia and G. Veneziano, Chiral dynamics in the large n limit, Nucl. Phys. B171, 253 (1980).

[11] A. V. Smilga, QCD at theta similar to pi, Phys. Rev. D 59, 114021 (1999).

[12] D. P. Horkel and S. R. Sharpe, Phase diagram of nondegenerate twisted mass fermions, Phys. Rev. D 90,
094508 (2014); Phase structure with nonzero $\Theta_{\mathrm{QCD}}$ and twisted mass fermions, Phys. Rev. D 92, 094514 (2015).

[13] M. Golterman and Y. Shamir, Effective potential in ultraviolet completions for composite Higgs models, Phys. Rev. D 97, 095005 (2018).

[14] J. Bijnens and J. Lu, Technicolor and other QCD-like theories at next-to-next-to-leading order, J. High Energy Phys. 11 (2009) 116.

[15] T. DeGrand, M. Golterman, E. T. Neil, and Y. Shamir, Oneloop Chiral perturbation theory with two fermion representations, Phys. Rev. D 94, 025020 (2016).

[16] M. Golterman, S. R. Sharpe, and R. L. Singleton, Jr., Effective theory for quenched lattice QCD and the Aoki phase, Phys. Rev. D 71, 094503 (2005).

[17] C.W. Bernard and M. Golterman, Chiral perturbation theory for the quenched approximation of QCD, Phys. Rev. D 46, 853 (1992).

[18] J. Smit, Fermions on a Lattice, Acta Phys. Pol. B 17, 531 (1986).

[19] H. Leutwyler and A. V. Smilga, Spectrum of Dirac operator and role of winding number in QCD, Phys. Rev. D 46, 5607 (1992).

[20] L.H. Karsten and J. Smit, Lattice Fermions: Species doubling, Chiral invariance, and the triangle anomaly, Nucl. Phys. B183, 103 (1981).

[21] S. Aoki, New phase structure for lattice QCD with Wilson Fermions, Phys. Rev. D 30, 2653 (1984).

[22] M. Bochicchio, L. Maiani, G. Martinelli, G. C. Rossi, and M. Testa, Chiral symmetry on the lattice with Wilson Fermions, Nucl. Phys. B262, 331 (1985). 